\title{
Florística e fitossociologia em três diferentes fitofisionomias campestres no Sul do Brasil ${ }^{1}$
}

\author{
Adriane Ribeiro da Silva ${ }^{2,4}$, Anna Luiza Pereira de Andrade ${ }^{2,3}$, Santiago Elías Velazco ${ }^{2}$, Franklin Galvão ${ }^{2}$ e \\ Marta Regina Barrotto do Carmo ${ }^{3}$
}

Recebido: 16.07.2015; aceito: 9.05.2016

\begin{abstract}
Floristic and phytosociology of three different grassland physiognomies of Southern Brazil). The purpose of this study was to evaluate the composition and structure of three different types of grassland vegetation of Southern Brazil. We aimed to discuss the floristic similarities among these areas and the influence of the Cerrado flora in their composition.

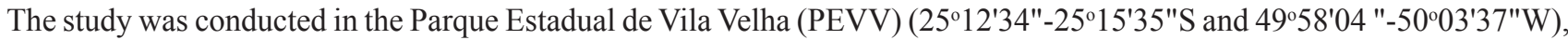
Municipality of Ponta Grossa, Paraná State, Brazil, in three grassland physiognomies: dry (CS), wet (CU) and rocky (CR). Thirty plots of $1 \times 1 \mathrm{~m}$ were placed in each area for the quantitative sampling. Additionally, species that occurred around the plots were also collected. In total, 283 species were sampled, with 163 species in CS, 87 species in CU, and 59 in CR. $36.48 \%$ (85) of the species sampled in this study also occur in the Cerrado. The cluster analysis showed that the floristic composition of the three grassland areas is significantly different due to several factors, especially the soil water system. Keywords: Campos Gerais, community structure, grassland, Paraná State, Southern Brazil
\end{abstract}

RESUMO - (Florística e fitossociologia em três diferentes fitofisionomias campestres no Sul do Brasil). A proposta deste estudo foi avaliar a composição e a estrutura de comunidades campestres, procurando discutir a similaridade florística entre as diferentes fitofisionomias e a influência da flora do Cerrado na sua composição. O estudo foi realizado no Parque Estadual

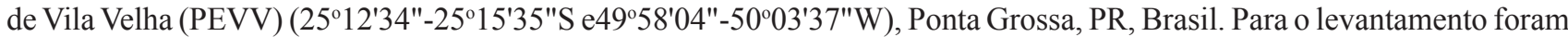
alocadas 30 parcelas de $1 \times 1 \mathrm{~m}$ em cada área,e acrescentadas coletas aleatórias nas fitofisionomias: campo seco (CS), campo úmido (CU) e campo rupestre (CR). Foram amostradas 283 espécies, sendo 163 espécies no CS, 87 no CU e 59 CR. Das espécies identificadas em nível específico,36,48\% (85) foram citadas para áreas de Cerrado da região. As análises de agrupamento apontaram que as composições florísticas das três formações campestres são significativamente distintas entre si, isto se deve a diversos fatores, especialmente em relação ao regime hídrico do solo.

Palavras-chave: Campos Gerais do Paraná, campos sulinos, estrutura de comunidade, vegetação campestre

\section{Introdução}

Os campos do Sul do Brasil são unidades fitogeográficas naturais que persistem na região após a expansão das formações florestais ocorrida na metade do Holoceno (Behling \& Pillar 2007, Dümig et al. 2008, Behling et al. 2009). Essas unidades são partes do bioma Pampa na porção sul e oeste do Rio Grande do Sul, e do bioma Mata Atlântica nas maiores altitudes do planalto do Sul do Brasil (IBGE 2012).
Originalmente a cobertura da vegetação campestre no Estado do Paraná era de 8,4\%, sendo encontrada em sua maior extensão na região denominada Campos Gerais (Maack 1948), situada na borda do Segundo Planalto. Os campos dessa região, apesar da aparente homogeneidade fisionômica, apresentam alta heterogeneidade florística (Moro \& Carmo 2007) e sofrem influência do Cerrado na sua composição (Carmo et al. 2012, Moro 2012). Tal região ainda mantem algumas áreas preservadas como

1. Parte da Dissertação de Mestrado da primeira Autora

2. Universidade Federal do Paraná, Pós-graduação em Engenharia Florestal, Av. Pref. Lothário Meissner, 900, Jardim Botânico, Campus III, 80210-170 Curitiba, PR, Brasil

3. Universidade Estadual de Ponta Grossa, Departamento de Biologia Geral, Av. Carlos Cavalcanti, 4.748, 84030-900, Ponta Grossa, PR, Brasil

4. Autor para correspondência: drica.rivers@gmail.com 
consequência da sua baixa aptidão agrícola, decorrente de solos pobres, rasos e arenosos, e principalmente pelo isolamento fitogeográfico imposto pela barreira geomorfológica representada pela Escarpa Devoniana (Melo et al. 2007).

Estes campos paranaenses apresentam predominância de cobertura herbácea (Klein \& Hatschbach 1971, Moro \& Carmo 2007), e podem ser agrupados em: (a) campos secos, encontrados em áreas bem drenadas, associados principalmente a Cambissolos, Argissolos, Neossolos Litóticos e Regolíticos de textura arenosa e média; (b) campos em afloramentos rochosos associados a Neossolos Litólicos, com vegetação sobre uma tênue camada de solo; (c) campos úmidos que ocupam pequenas extensões onde há acúmulo de água, por vezes próximo a córregos, outras em manchas onde o nível do lençol freático é superficial. Nessas condições, os campos ocorrem em Gleissolos e Organossolos, ambos solos hidromórficos, marcados pela presença de espécies poupadas do fogo devido à umidade constante do terreno (Moro \& Carmo 2007).

Assim como as demais regiões no Sul do Brasil, os Campos Gerais têm sido alvo da ocupação humana e de atividades agropecuárias, e vem sofrendo alterações de maneira sistemática desde o século XVIII, com a colonização de paulistas, a intensificação da imigração europeia (Moro 2001) e a contaminação biológica por espécies florestais exóticas (Ziller \& Galvão 2002).

Trabalhos na região dos Campos Gerais do Paraná foram realizados a partir da década 1970 (Klein \& Hatschbach 1971, Hatschbach \& Moreira Filho 1972), mas somente nos últimos anos pesquisadores têm somado esforços para caracterizar a flora campestre da região (Moro et al. 1996, Cervi et al. 2007, Moro \& Carmo 2007, Dalazoana et al. 2007, Kozera et al. 2009, Andrade et al. 2011, Dalazoana \& Moro 2011, Moro et al. 2012). Estes estudos têm reforçado o caráter frágil desta unidade pela natureza de seus solos e pelo efeito deletério à riqueza florística provocado pelos plantios agrícolas, pecuária e atividades silviculturais recentes (Ramos et al. 2007). Além disso, na região foram encontradas espécies consideradas raras e/ou endêmicas, incluídas na Lista Oficial de Espécies Ameaçadas de Extinção no Estado do Paraná (Moro \& Carmo 2007, Kozera et al. 2009, Carmo et al. 2012). Porém, trabalhos sobre a estrutura e dinâmica das comunidades campestres ainda são considerados insuficientes (Moro \& Carmo 2007).

Portanto, estudos adicionais na região são necessários, para somar esforços no entendimento da complexidade desta unidade fitogeográfica e sinalizar, de maneira consistente, estratégias corretas para a sua conservação. Neste sentido, a proposta deste estudo foi avaliar a composição e a estrutura de comunidades campestres em uma Unidade de Conservação da região dos Campos Gerais do Paraná, procurando discutir a similaridade florística entre as diferentes fitofisionomias e a influência da flora do cerrado na sua composição.

\section{Material e métodos}

Área de Estudo - O estudo foi realizado no Parque Estadual de Vila Velha (PEVV) $\left(25^{\circ} 12^{\prime} 34^{\prime \prime}\right.$ e $25^{\circ} 15^{\prime} 35^{\prime \prime} \mathrm{S}$ e $49^{\circ} 58^{\prime} 04^{\prime \prime}$ e $50^{\circ} 03^{\prime} 37^{\prime \prime} \mathrm{W}$ - altitude de 800 a $1.068 \mathrm{~m}$ ) uma Unidade de Conservação de 3.803 ha localizada no município de Ponta Grossa, região dos Campos Gerais do Paraná (figura 1).

O clima no PEVV, segundo a classificação de Köeppen (1948), é do tipo Cfb, com média anual de $1.554 \mathrm{~mm}$ de precipitação (Caramari 2004). Os meses de janeiro e fevereiro são os mais quentes do ano, com temperatura média mensal de $21,4{ }^{\circ} \mathrm{C}$. O mês mais frio do ano é julho, apresentando média mensal de $13,8^{\circ} \mathrm{C}$. As geadas concentram-se entre os meses de maio e agosto, tendo em média mais de cinco geadas por ano (Melo 2006). O mês de janeiro é o mais chuvoso, totalizando média de $168 \mathrm{~mm}$, e o mês de agosto é o mais seco do ano, com precipitação média de 78 mm (Caramari 2004). Embora ocorram dias longos no verão, o número de horas de insolação é relativamente pequeno, não ultrapassando sete horas diárias em média. Isto ocorre devido à grande nebulosidade em consequência da elevada umidade do ar (Caramari 2004).

As rochas que ocorrem dentro do perímetro do PEVV e proximidades pertencem principalmente às unidades mais antigas da Bacia do Paraná: formações Furnas e Ponta Grossa (Devoniano) e Grupo Itararé (Carbonífero - Permiano) (Melo 2006). A heterogeneidade geomorfológica leva à diversidade de compartimentos geopedológicos sendo representado pelos: Latossolos Vermelhos, Latossolos VermelhoAmarelos, Cambissolos Húmicos, Cambissolos Háplicos, Neossolos Litólicos, Neossolos Flúvicos, Neossolos Quartzarênicos, Gleissolos e Organossolos (Curcio et al. 2004).

O PEVV é uma das maiores Unidades de Conservação do Estado do Paraná, com relictos de vegetação campestre, abrangendo também áreas de Floresta Ombrófila Mista, que aparecem na forma 
de capões e ao longo dos cursos d'água. No parque são identificadas três formações campestres: campo seco, onde os solos são bem drenados, de textura arenosa, com 185 ha, entre 880 e 900 m a.n.m. de altitude; campo úmido que está condicionado ao encharcamento constante dos solos, área de 65 ha com profundidade média de um metro, onde o relevo é suave-ondulado, em altitude de $800 \mathrm{~m}$ a.n.m.; e o campo rupestre, onde a vegetação se desenvolve sobre uma tênue camada de solo, possui uma extensão de 72 ha, em altitudes de 1000 a 1015 m a.n.m.

Nestas fitofisionomias campestres do PEVV ocorrem queimadas esporádicas e acidentais, como foram observados três meses que antecederam o início desta pesquisa (novembro de 2011) nas áreas de campo seco e campo úmido. As frequências das queimadas do PEVV são geralmente anuais ou em intervalos maiores, sendo que em tais áreas, até a execução deste estudo, não havia atividades de manejo com o uso de fogo controlado.

Metodologia - O levantamento foi realizado em três fitofisionomias campestres, compreendendo áreas

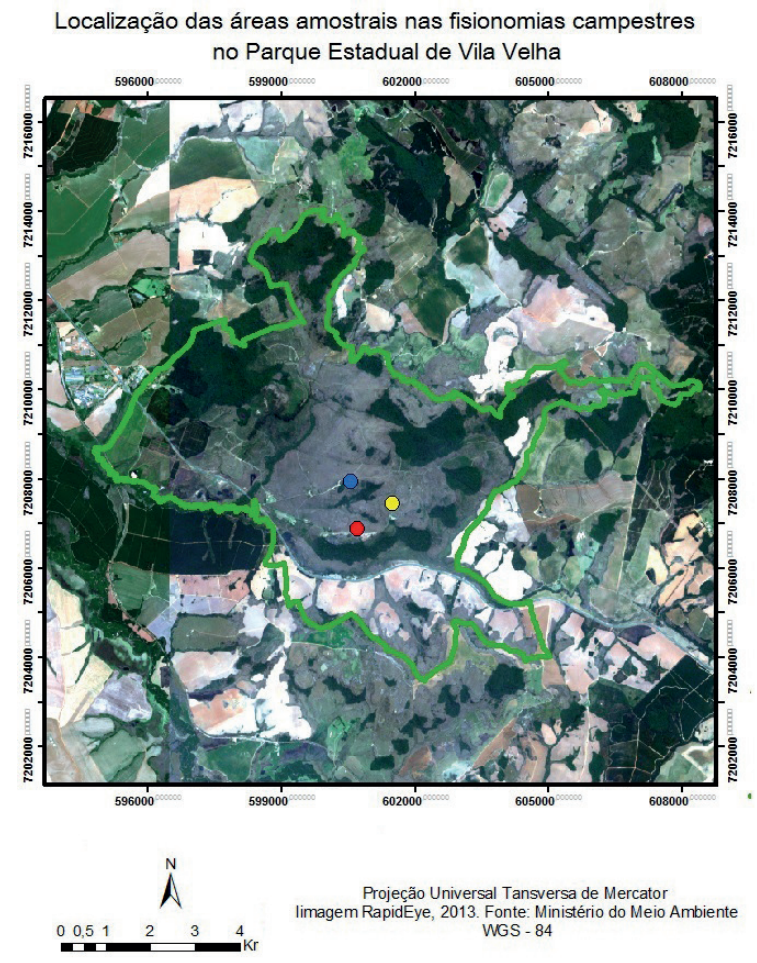

Figura 1. Localização das áreas amostradas no Parque Estadual de Vila Velha, Ponta Grossa, Paraná. Áreas: CR: Campo rupestre. - CS: Campo seco, ○ CU: Campo úmido,_Limite do Parque Estadual de Vila Velha

Figure 1. Localization of the areas sampled in the Vila Velha State Park, Municipality of Ponta Grossa, Paraná State, Brazil. Areas: CR: Rocky grassland. C CS: Dry grassland, CU: Wet grassland, Limits of Parque Estadual de Vila Velha. de campo seco (CS), campo úmido (CU) e campo rupestre (CR) (figura 1).

Para o estudo fitossociológico foram alocadas, em cada área, 30 parcelas de $1 \times 1 \mathrm{~m}$, dispostas em três linhas de cinco blocos de duas parcelas contíguas, com distância sempre de 10 metros entre linhas e blocos, totalizando $90 \mathrm{~m}^{2}$ de área de amostragem. No CR, as parcelas foram alocadas estritamente nas fraturas das rochas onde havia acúmulo de solo e propiciava desenvolvimento de vegetação.

Dentro das parcelas, após a identificação dos espécimes, foram estimadas as coberturas de cada espécie, segundo a escala de Daubenmire (1968): 1 (0-5\%), 2 (5-25\%), 3 (25-50\%), 4 (50-75\%), 5 (75-95\%), 6 (95-100\%). Os descritores quantitativos avaliados foram: presença $(\mathrm{Pi})$, frequência relativa (FR), cobertura relativa (CR) e valor de importância (VI) (Daubenmire 1968).

Para o estudo florístico foram também acrescentadas nas três fitofisionomias coletas aleatórias de espécies vasculares em estágio reprodutivo, através de caminhadas de aproximadamente $50 \mathrm{~m}$ de distância das parcelas.

Todo material botânico em fase reprodutiva foi herborizado (Fidalgo \& Bononi 1984) e posteriormente identificado por comparação com exsicatas de herbários e por consulta à bibliografia especializada. Os exemplares coletados foram incorporados ao acervo do Herbário da Universidade Estadual de Ponta Grossa (HUPG). A grafia do nome das espécies e de seus autores foi atualizada através de consultas à base de dados do programa REFLORA (Flora do Brasil 2020), seguindo o sistema de classificação APG III (2009) para fanerógamas e Pichi-Sermolli (1996) para pteridófitas.

A exaustão amostral foi avaliada por meio da comparação da riqueza de espécies encontradas nas parcelas e a quantidade de espécies preditas pelo estimador de riqueza não-paramétrico Chao 2. A riqueza de cada fitofisionomia campestre foi comparada através de curvas de rarefação e por bandas de confiança geradas por 100 aleatorizações das unidades amostrais. Estas análises foram geradas no programa EstimateS 9.1.0 (Colwell 2013).

A similaridade florística foi calculada por meio do índice de similaridade de Sorensen, empregando uma matriz onde as colunas $\mathrm{Xj}$ representavam as espécies, as linhas $\mathrm{Xi}$ as unidades amostrais e cada valor $\mathrm{Xij}$ a presença/ausência de cada espécie para cada parcela. Esta matriz de similaridade foi empregada para classificar as unidades amostrais por meio de 
uma análise de agrupamento por médias aritméticas não ponderadas (Unweighted arithmetic average clustering - UPGMA). A qualidade do agrupamento foi avaliada por meio da correlação cofenética, valores de correlação mais próximos a um indicam um melhor modelo de agrupamento. Os três grupos gerados na análise de agrupamento foram testados por meio do procedimento de permutação multi-resposta (Multi Response Permutation Procedure - MRPP) com 999 permutações e o índice de Sorensen. De maneira a diminuir a multidimensionalidade da matriz de espécies e achar um padrão das unidades amostrais, foi realizada uma análise de ordenação non-metric multidimensional scaling (NMDS) com distância de Sorensen e 100 interações, selecionando duas dimensões. O desempenho desta análise foi determinado pelo valor do estresse. Assim, valores $<$ a 0,05 denotam uma ordenação quase excelente, enquanto que valores $>$ a 0,3 são considerados desempenho muito baixo (Zuur et al. 2007). Os eixos de ordenação foram testados por meio do teste de Mantel com 999 permutações. As análises multivariadas e os testes MRPP e Mantel foram efetuados no R v. 3.2.2 (R Core Team 2015), usando os pacotes vegan e cluster. Os gráficos foram elaborados com o pacote ggplot2.

Para verificar a influência da flora do Cerrado na composição florística dos campos do PEVV, foram analisadas as listas de espécies de trabalhos realizados em áreas comprovadamente de Cerrado da região (Ritter et al. 2007, Cervi et al. 2007, Carmo et al. 2012, Moro et al. 2012).

\section{Resultados}

Florística - Foram amostradas 283 espécies (tabela 1) compreendidas em 55 famílias, e mais nove morfoespécies indeterminadas a qualquer nível taxonômico, devido ao seu estágio vegetativo ou de plântula. Para o campo seco (CS) foram amostradas 163 espécies, seguida de 87 no campo úmido (CU) e 59 no campo rupestre (CR).

As famílias com maior riqueza florística foram Asteraceae (74 espécies), seguida de Poaceae (33), Cyperaceae (21), Fabaceae (20) e Melastomataceae (10), totalizando $55,8 \%$ das espécies amostradas. As demais espécies foram distribuídas em 49 famílias, sendo que $51,02 \%$ destas foram representadas por uma única espécie (tabela 1).

No campo seco, as famílias mais representativas foram Asteraceae (48), Poaceae (19) e Fabaceae
(17), que juntas representaram 51,53\% das espécies amostradas nesta fisionomia. No campo úmido, as famílias com maior riqueza florística foram Asteraceae (21), Cyperaceae (16) e Poaceae (10), totalizando $54,02 \%$ das espécies amostradas, enquanto que no campo rupestre as mais representativas foram Asteraceae (12), Poaceae (10) e Bromeliaceae (4), representando $44,06 \%$ do total de espécies amostradas.

Sete famílias foram comuns as três fitofisionomias: Apocynaceae, Asteraceae, Cyperaceae, Fabaceae, Iridaceae, Melastomataceae e Poaceae. As famílias exclusivas no CS foram Aquifoliaceae, Bignoniaceae, Boraginaceae, Campanulaceae, Celastraceae, Convolvulaceae, Cucurbitaceae, Erythroxylaceae, Hypericaceae, Lythraceae, Malpighiaceae, Malvaceae, Oxalidaceae, Turneraceae e Dennstaedtiaceae. No CU as famílias exclusivas foram Begoniaceae, Lentibulariaceae, Onagraceae, Xyridaceae, Thelypteridaceae, Blechnaceae e Cyatheaceae. Já para o CR foram Amaryllidaceae, Bromeliaceae, Commelinaceae, Ericaceae, Primulaceae, Portulacaceae, Rhamnaceae, Solanaceae e Polypodiaceae.

Somente três espécies foram comuns às fitofisionomias estudadas, sendo elas Bulbostylis capillaris, Achyrocline satureioides e Andropogon leucostachyus. Consequentemente, a similaridade entre as três fisionomias se mostrou baixa. $\mathrm{O}$ índice de Sorensen foi de 6,30\% entre o CS e CR, onde as espécies comuns foram Stevia leptophylla, Allagoptera campestris, Cinnamomum sellowianum, Parodia carambeiensis, Galactia neesii, Leandra aurea e Trachypogon spicatus. Entre o CS e CU foi de $8 \%$, tendo como espécies comuns Baccharis illinita, Chromolaena laevigata, Chromolaena sp. 1, Croton antisyphiliticus, Croton splendidus, Dichanthelium sabulorum, Sisyrinchium vaginatum, Polygala longicaulis e Galium megapotamicum. As fitofisionomias que obtiveram a menor similaridade foram CU e CR, com 4,10\%, tendo como espécies comuns Drosera communis, Paepalanthus albovaginatus e Oxypetalum sublanatum.

As curvas de rarefação mostraram diferentes tendências assintóticas para cada comunidade (figura 2). O CR foi o de menor exaustão amostral, seguida do CS e do CU. Assim, considerando só as espécies dentro das parcelas, estas representaram $72 \%$, $77 \%$ e $89 \%$ da riqueza estimada pelo Chao 2 para o $\mathrm{CR}, \mathrm{CS}$ e CU, respectivamente. Mas, ao considerar as espécies coletadas fora das unidades amostrais, estas superam as riquezas estimadas. A não sobreposição 
Tabela 1. Relação das espécies amostradas nas fitofisionomias campestres do Parque Estadual de Vila Velha, Ponta Grossa, Paraná. CS: Campo seco; CU: Campo úmido; CR: Campo rupestre; HUPG: Número tombo no Herbário da Universidade Estadual de Ponta Grossa. * Espécies citadas para a flora do Cerrado.

Table 1. List of species sampled in grassland physiognomies, State Park of Vila Velha, Municipality of Ponta Grossa, Paraná State, Brazil. CS: Dry grassland; CU: Wet grassland; CR: Rocky grassland; HUPG: Voucher number in the Herbarium of the State University of Ponta Grossa. * Cerrado species.

\begin{tabular}{|c|c|c|}
\hline Família/Espécies & Campo & HUPG \\
\hline \multicolumn{3}{|l|}{ Amaranthaceae } \\
\hline Alternanthera brasiliana (L.) Kuntze* & $\mathrm{CS}$ & 20394 \\
\hline Gomphrena paranensis R.E. Fr. & $\mathrm{CS}$ & 20214 \\
\hline Pfaffia gnaphaloides (L.f.) Mart. & $\mathrm{CU}$ & 20266 \\
\hline Pfaffia helichrysoides (Mart.) Kuntze & $\mathrm{CS}$ & \\
\hline \multicolumn{3}{|l|}{ Amaryllidaceae } \\
\hline Hippeastrum psittacinum Herb.* & $\mathrm{CR}$ & 20282 \\
\hline \multicolumn{3}{|l|}{ Apiaceae } \\
\hline Eryngium ebracteatum Lam.* & $\mathrm{CU}$ & 20115 \\
\hline Eryngium elegans Cham. \& Schltdl.* & $\mathrm{CS}$ & 20352 \\
\hline Eryngium horridum Malme* & $\mathrm{CS}$ & 20164 \\
\hline Eryngium junceum Cham. \& Schltdl.* & $\mathrm{CU}$ & 20345 \\
\hline Eryngium sanguisorba Cham. \& Schltdl.* & $\mathrm{CS}$ & 20200 \\
\hline \multicolumn{3}{|l|}{ Apocynaceae } \\
\hline Mandevilla coccinea (Hook. \& Arn.) Woodson* & $\mathrm{CS}$ & 20382 \\
\hline Mandevilla pohliana (Stadelm.) A.H. Gentry & $\mathrm{CS}$ & 20319 \\
\hline Oxypetalum sublanatum Malme* & $\mathrm{CU} / \mathrm{CR}$ & 20258 \\
\hline \multicolumn{3}{|l|}{ Aquifoliaceae } \\
\hline Ilex chamaedryfolia Reissek & $\mathrm{CS}$ & 20123 \\
\hline \multicolumn{3}{|l|}{ Arecaceae } \\
\hline Allagoptera campestris (Mart.) Kuntze* & $\mathrm{CS} / \mathrm{CR}$ & 20215 \\
\hline Butia microspadix Burret & CS & 20216 \\
\hline Syagrus romanzoffiana (Cham.) Glassman* & $\mathrm{CR}$ & \\
\hline \multicolumn{3}{|l|}{ Asteraceae } \\
\hline Achyrocline satureioides (Lam.) DC.* & $\mathrm{CS} / \mathrm{CU} / \mathrm{CR}$ & 20371 \\
\hline Acitepidopsis echitifolia (Mart. ex Dc.) H. Rob. & $\mathrm{CU}$ & 20213 \\
\hline Aldama kunthiana (Gardner) E. E. Schil. \& Panero & $\mathrm{CS}$ & 20203 \\
\hline Aspilia montevidensis (Spreng.) Kuntze & $\mathrm{CU}$ & 20304 \\
\hline Austroeupatorium inulaefolium (Kunth) R.M King \& H. Rob* & $\mathrm{CU}$ & 20377 \\
\hline Baccharis axillaris DC. & $\mathrm{CR}$ & 20104 \\
\hline Baccharis coridifolia DC. & $\mathrm{CS}$ & 20153 \\
\hline Baccharis crispa Spreng & $\mathrm{CU}$ & 20260 \\
\hline Baccharis illinita DC. & $\mathrm{CS} / \mathrm{CU}$ & \\
\hline Baccharis myricifolia DC. & $\mathrm{CS}$ & 20126 \\
\hline Baccharis reticularia DC. & $\mathrm{CR}$ & 20362 \\
\hline Baccharis sessilliflora Vahl. & $\mathrm{CS}$ & 20369 \\
\hline Baccharis tarchonanthoides DC. & $\mathrm{CR}$ & 20380 \\
\hline Baccharis sp.1 & $\mathrm{CS}$ & 20167 \\
\hline
\end{tabular}


Tabela 1 (continuação)

\begin{tabular}{|c|c|c|}
\hline Família/Espécies & Campo & HUPG \\
\hline Calea cymosa Less. & $\mathrm{CS}$ & 20169 \\
\hline Calea parvifolia (DC.) Baker & $\mathrm{CR}$ & 20107 \\
\hline Campovassouria cruciata (Vell.) R.M. King \& H. Rob. & $\mathrm{CU}$ & 20375 \\
\hline Cantinoa althaeifolia (Pohl ex Benth.) Harley \& J.F.B. Pastore & $\mathrm{CU}$ & 20098 \\
\hline Chaptalia integerrima (Vell.) Burkart* & $\mathrm{CS}$ & 20328 \\
\hline Chromolaena congesta (Hook. \& Arn.) R.M. King \& H. Rob. & $\mathrm{CR}$ & 20109 \\
\hline Chromolaena laevigata (Lam.) R.M. King \& H. Rob* & $\mathrm{CS} / \mathrm{CU}$ & 20356 \\
\hline Chromolaena palmaris (Sch.Bip. ex Baker) R.M. King \& H. Rob. & CS & 20196 \\
\hline Chromolaena sp. 1 & $\mathrm{CS} / \mathrm{CU}$ & 20376 \\
\hline Chrysolaena platensis (Spreng.) H. Rob.* & $\mathrm{CS}$ & 20158 \\
\hline Chrysolaena simplex (Less.) Dematt.* & $\mathrm{CS} / \mathrm{CU}$ & 20331 \\
\hline Conyza bonariensis (L.) Cronquist & CR & 20400 \\
\hline Elephantopus sp. 1 & $\mathrm{CS}$ & 20307 \\
\hline Erechtites valerianifolius (Wolf) DC.* & $\mathrm{CU}$ & 20359 \\
\hline Eupatorieae sp. 1 & $\mathrm{CS}$ & \\
\hline Eupatorieae sp. 2 & $\mathrm{CS}$ & 20179 \\
\hline Gamochaeta purpurea (L.) Cabrera* & $\mathrm{CU}$ & 20311 \\
\hline Gochnatia argyrea (Dusén ex Malme) Cabrera & $\mathrm{CS}$ & 20353 \\
\hline Grazielia intermedia (DC.) R.M. King \& H. Rob.* & $\mathrm{CU}$ & 20211 \\
\hline Grazielia multifida (Dc.) R. M. King. \& H. Rob & $\mathrm{CS}$ & 20368 \\
\hline Gyptis lanigera (Hook. \& Arn) R. M. King \& H. Rob & $\mathrm{CS}$ & 20174 \\
\hline Gyptis pinnatifita Cass.* & $\mathrm{CS}$ & 20269 \\
\hline Heterocondylus pumilus (Gardner) R.M. King \& H. Rob. & $\mathrm{CS}$ & 20372 \\
\hline Hypochaeris lutea (Vell.) Britton & $\mathrm{CS}$ & 20294 \\
\hline Isostigma speciosum Less. & $\mathrm{CS}$ & 20193 \\
\hline Lessingianthus asteriflorus (Mart. ex DC.) H. Rob. & $\mathrm{CS}$ & 20180 \\
\hline Lessingianthus brevifolius (Less.) H. Rob. & $\mathrm{CS}$ & 20187 \\
\hline Lessingianthus grandiflorus (Less.) H. Rob. & $\mathrm{CS}$ & 20114 \\
\hline Lessingianthus rubricaulis (Humb. \& Bonpl.) H. Rob. & $\mathrm{CS}$ & 20177 \\
\hline Lessingianthus sp. & $\mathrm{CS}$ & 20283 \\
\hline Lucilia acutifolia (Poir.) Cass. & $\mathrm{CS}$ & 20289 \\
\hline Lucilia lycopodioides (Less.) S.E. Freire & $\mathrm{CS}$ & 20157 \\
\hline Mikania micrantha Kunth & $\mathrm{CU}$ & 20240 \\
\hline Mikania oblongifolia DC.* & $\mathrm{CS}$ & 20351 \\
\hline Praxelis sanctopaulensis (B.L. Rob.) R.M. King \& H. Rob. & $\mathrm{CR}$ & 20221 \\
\hline Pterocaulon angustifolium DC. & $\mathrm{CS}$ & 20186 \\
\hline Pterocaulon balansae Chodat. & $\mathrm{CU}$ & 20124 \\
\hline Senecio leptoschizus Bong. & $\mathrm{CS}$ & 20370 \\
\hline Senecio oleosus Vell. & $\mathrm{CS}$ & 20171 \\
\hline Stenocephalum megapotamicum (Spreng.) Sch.Bip. & $\mathrm{CS}$ & 20176 \\
\hline Stevia leptophylla Sch.Bip. ex Baker & $\mathrm{CS} / \mathrm{CR}$ & 20366 \\
\hline Symphyopappus cuneatus (DC.) Sch. Bip. ex Baker & $\mathrm{CR}$ & 20338 \\
\hline Trichocline speciosa Less. & $\mathrm{CS}$ & 20195 \\
\hline
\end{tabular}


Tabela 1 (continuação)

\begin{tabular}{|c|c|c|}
\hline Família/Espécies & Campo & HUPG \\
\hline Verbesina sordescens DC. & $\mathrm{CS}$ & 20172 \\
\hline Vernonanthura crassa (Vell.) H. Rob. & $\mathrm{CR}$ & 20120 \\
\hline Vernonanthura cuneifolia (Gardner) H. Rob.* & $\mathrm{CR}$ & 20118 \\
\hline Vernonanthura nudiflora (Less.) H. Rob & $\mathrm{CS}$ & \\
\hline Vernonanthura sp. 1 & $\mathrm{CS}$ & \\
\hline Vittetia orbiculata (DC.) R.M. King \& H. Rob. & $\mathrm{CS}$ & 20108 \\
\hline Asteraceae 1 & $\mathrm{CS}$ & \\
\hline Asteraceae 2 & $\mathrm{CS}$ & \\
\hline Asteraceae 3 & $\mathrm{CU}$ & 20347 \\
\hline Asteraceae 4 & $\mathrm{CS}$ & \\
\hline Asteraceae 5 & $\mathrm{CU}$ & 20212 \\
\hline Asteraceae 6 & $\mathrm{CU}$ & \\
\hline Asteraceae 7 & $\mathrm{CU}$ & \\
\hline Asteraceae 8 & $\mathrm{CS}$ & \\
\hline Asteraceae 9 & $\mathrm{CU}$ & \\
\hline Asteraceae 10 & $\mathrm{CS}$ & \\
\hline \multicolumn{3}{|l|}{ Begoniaceae } \\
\hline Begonia fischeri Schrank* & $\mathrm{CU}$ & 20333 \\
\hline \multicolumn{3}{|l|}{ Bignoniaceae } \\
\hline Jacaranda caroba (Vell.) DC.* & $\mathrm{CS}$ & 20162 \\
\hline \multicolumn{3}{|l|}{ Blechnaceae } \\
\hline Blechnum cordatum (Desv.) Hieron. & $\mathrm{CU}$ & \\
\hline \multicolumn{3}{|l|}{ Boraginaceae } \\
\hline Moritzia dusenii I.M. Johnst & $\mathrm{CS}$ & 20298 \\
\hline \multicolumn{3}{|l|}{ Bromeliaceae } \\
\hline Aechmea distichantha Lem.* & $\mathrm{CR}$ & 20254 \\
\hline Tillandsia crocata (E. Morren) Baker & $\mathrm{CR}$ & 20113 \\
\hline Tillandsia stricta Sol.* & $\mathrm{CR}$ & 20383 \\
\hline Vriesea friburgensis Mez* & $\mathrm{CR}$ & \\
\hline \multicolumn{3}{|l|}{ Cactaceae } \\
\hline Parodia carambeiensis Buining \& Brederoo & $\mathrm{CS} / \mathrm{CR}$ & \\
\hline Rhipsalis neves-armondii K. Schum. & $\mathrm{CR}$ & \\
\hline \multicolumn{3}{|l|}{ Campanulaceae } \\
\hline Lobelia camporum Poh1* & $\mathrm{CS}$ & 20121 \\
\hline \multicolumn{3}{|l|}{ Celastraceae } \\
\hline Plenckia populnea Reissek* & $\mathrm{CS}$ & 20286 \\
\hline \multicolumn{3}{|l|}{ Commelinaceae } \\
\hline Commelina villosa C. B. Clarke ex Chodat \& Hassl. & $\mathrm{CR}$ & \\
\hline \multicolumn{3}{|l|}{ Convolvulaceae } \\
\hline Evolvulus alsinoides $\mathrm{L}$. & $\mathrm{CS}$ & 20336 \\
\hline Evolvulus sericeus Sw. var. sericeus & $\mathrm{CS}$ & 20151 \\
\hline Ipomoea delphinioides Choisy & $\mathrm{CS}$ & 20384 \\
\hline Convolvulaceae 1 & $\mathrm{CS}$ & 20284 \\
\hline
\end{tabular}


Tabela 1 (continuação)

Família/Espécies

Campo

HUPG

Cucurbitaceae

Cayaponia espelina (Silva Manso) Cogn.

CS

20190

Cyatheaceae

Cyathea atrovirens (Langsd. \& Fisch.) Domin

$\mathrm{CU}$

Cyperaceae

Ascolepis brasiliensis (Kunth) Benth. ex C.B. Clarke

$\mathrm{CU}$

20302

Bulbostylis capillaris (L.) C.B. Clarke*

$\mathrm{CS} / \mathrm{CU} / \mathrm{CR}$

20208

Bulbostylis juncoides (Vahl) Kük.

$\mathrm{CU}$

20346

Bulbostylis scabra (J.Presl \& C.Presl) C.B.Clarke

CS

20337

Bulbostylis sp. 1

$\mathrm{CU}$

20233

Bulbostylis sphaerocephala (Boeckeler) C.B. Clarke

CS

20334

Cyperus aggregatus (Willd.) Endl.

CR

20373

Cyperus intricatus Schrad. ex Schult.

$\mathrm{CU}$

20301

Cyperus rotundus L.*

$\mathrm{CU}$

20239

Pycreus lanceolatus (Poir.) C.B. Clarke

$\mathrm{CU}$

20237

Pycreus polystachyos (Rottb.) P. Beauv.

$\mathrm{CU}$

20232

Rhynchospora albiceps Kunth

$\mathrm{CU}$

20247

Rhynchospora globosa (Kunth) Roem. \& Schult.*

$\mathrm{CU}$

20100

Rhynchospora riparia (Nees) Boeckeler*

$\mathrm{CU}$

20253

Rhynchospora rugosa (Vahl) Gale

$\mathrm{CU}$

20342

Rhynchospora terminalis Nees ex Steud.*

CR

20281

Rhynchospora sp.1

$\mathrm{CU}$

Scleria hirtella Sw.*

$\mathrm{CU}$

20255

Cyperaceae 1

$\mathrm{CU}$

20243

Cyperaceae 2

$\mathrm{CU}$

20117

Dennstaedtiaceae

Pteridium arachnoideum (Kaufl.) Maxon

CS

Droseraceae

Drosera communis A. St.-Hil

$\mathrm{CU} / \mathrm{CR}$

20252

Ericaceae

Gaylussacia brasiliensis (Spreng.) Meisn.

CR

20358

Eriocaulaceae

Eriocaulon ligulatum (Vell.) L.B.Sm.

Eriocaulon sellowianum Kunth

CR

20256

Paepalanthus albo-vaginatus Silveira

$\mathrm{CU}$

20300

$\mathrm{CU} / \mathrm{CR}$

20248

Paepalanthus caldensis Malme

$\mathrm{CU}$

20229

Syngonanthus caulescens (Poir.) Ruhland*

$\mathrm{CU}$

20249

Erythroxylaceae

Erythroxylum microphyllum A. St.-Hil.

CS

20293

Euphorbiaceae

Croton antisyphiliticus Mart.

$\mathrm{CS} / \mathrm{CU}$

20192

Croton heterodoxus Baill.

CS

20102

Croton solanaceus (Müll. Arg.) G.L. Webster

CS

20262

Croton splendidus Mart.

$\mathrm{CS} / \mathrm{CU}$

20381 
Tabela 1 (continuação)

\begin{tabular}{|c|c|c|}
\hline Família/Espécies & Campo & HUPG \\
\hline \multicolumn{3}{|l|}{ Fabaceae } \\
\hline Chamaecrista punctata (Vogel) H.S. Irwin \& Barneby & $\mathrm{CS}$ & 20152 \\
\hline Chamaecrista ramosa (Vogel) H.S. Irwin \& Barneby* & $\mathrm{CS}$ & 20354 \\
\hline Chamaecrista rotundifolia (Pers.) Greene & $\mathrm{CU}$ & 20217 \\
\hline Chamaecrista vestita (Vogel) H.S.Irwin \& Barneby & $\mathrm{CS}$ & 20197 \\
\hline Crotalaria balansae Micheli & $\mathrm{CS}$ & \\
\hline Desmodium barbatum (L.) Benth.* & $\mathrm{CU}$ & 20219 \\
\hline Desmodium incanum DC. & $\mathrm{CS}$ & \\
\hline Eriosema glabrum Mart. ex Benth.* & $\mathrm{CS}$ & 20165 \\
\hline Eriosema heterophyllum Benth.* & $\mathrm{CS}$ & \\
\hline Eriosema longifolium Benth.* & $\mathrm{CS}$ & 20116 \\
\hline Galactia neesii DC.* & $\mathrm{CS} / \mathrm{CR}$ & 20103 \\
\hline Rhynchosia corylifolia Mart. ex Benth. & CS & 20201 \\
\hline Stylosanthes bracteata Vogel* & $\mathrm{CS}$ & 20207 \\
\hline Stylosanthes guianensis (Aubl.) Sw.* & $\mathrm{CS}$ & 20119 \\
\hline Stylosanthes hippocampoides Mohlenbr. & $\mathrm{CS}$ & 20183 \\
\hline Stylosanthes montevidensis Vogel & $\mathrm{CS}$ & 20168 \\
\hline Vigna peduncularis (Kunth) Fawc. \& Rendle* & $\mathrm{CU}$ & 20305 \\
\hline Zornia cryptantha Arechav. & $\mathrm{CS}$ & 20184 \\
\hline Zornia diphylla (L.) Pers. & $\mathrm{CS}$ & 20318 \\
\hline Fabaceae 1 & $\mathrm{CS}$ & 20397 \\
\hline \multicolumn{3}{|l|}{ Gesneriaceae } \\
\hline Sinningia allagophylla (Mart.) Wiehler* & $\mathrm{CS}$ & 20378 \\
\hline Sinningia canescens (Mart.) Wiehler & $\mathrm{CR}$ & 20303 \\
\hline Sinningia elatior (Kunth) Chautems* & $\mathrm{CU}$ & \\
\hline \multicolumn{3}{|l|}{ Hypericaceae } \\
\hline Hypericum cordatum (Vell.) N.Robson & $\mathrm{CS}$ & 20170 \\
\hline Hypericum pirai Arechav & $\mathrm{CS}$ & 20310 \\
\hline \multicolumn{3}{|l|}{ Iridaceae } \\
\hline Calydorea campestris (Klatt) Baker & $\mathrm{CU}$ & 20288 \\
\hline Sisyrinchium restioides Spreng.* & CS & 20189 \\
\hline Sisyrinchium vaginatum Spreng. & $\mathrm{CS} / \mathrm{CU}$ & 20188 \\
\hline Sisyrinchium wettsteinii Hand.-Mazz. & $\mathrm{CR}$ & 20226 \\
\hline \multicolumn{3}{|l|}{ Lamiaceae } \\
\hline Aegiphila verticillata Vell.* & $\mathrm{CR}$ & 20314 \\
\hline Hyptis althaeifolia Pohl ex Benth. & $\mathrm{CR}$ & 20270 \\
\hline Peltodon longipes A. St.-Hil. ex Benth. & $\mathrm{CS}$ & 20191 \\
\hline Salvia aliciae E.P. Santos & $\mathrm{CS}$ & \\
\hline Salvia sp.1 & $\mathrm{CS}$ & 20267 \\
\hline Vitex megapotamica (Spreng.) Moldenke* & $\mathrm{CR}$ & \\
\hline \multicolumn{3}{|l|}{ Lauraceae } \\
\hline Cinnamomum sellowianum (Nees \& Mart.) Kosterm. & $\mathrm{CS} / \mathrm{CR}$ & 20387 \\
\hline
\end{tabular}


Tabela 1 (continuação)

Família/Espécies Campo

HUPG

Lentibulariaceae

Utricularia praelonga A. St.-Hil. \& Girard*

$\mathrm{CU}$

20250

Lythraceae

Cuphea linifolia (A. St.-Hil.) Koehne

CS

20321

Malpighiaceae

Aspicarpa pulchella (Griseb.) O’ Donell \& Lourteig*

CS

20344

Byrsonima brachybotrya Nied.

20111

Byrsonima guilleminiana A. Juss.*

20239

Heteropterys dusenii Nied.

20396

Malvaceae

Byttneria hatschbachii Cristóbal

Krapovickasia macrodon (A. DC.) Fryxell*

CS

Peltaea speciosa (Kunth) Standl.

$\mathrm{CS}$

CS

Sida sp.

Waltheria communis A. St.-Hil.*

$\mathrm{CS}$

20105

$\mathrm{CS}$

20154

$\mathrm{CS}$

20095

Melastomataceae

Acisanthera quadrata Pers.*

$\mathrm{CU}$

20155

Lavoisiera pulchella Cham.*

$\mathrm{CU}$

20150

Leandra erostrata (DC.) Cogn.

20245

Leandra polystachya (Naudin) Cogn.*

CS

20279

Leandra aurea (Cham.) Cogn.

$\mathrm{CS}$

20246

Miconia hyemalis A. St.-Hil et Naudin

$\mathrm{CS} / \mathrm{CR}$

20306

Tibouchina debilis Cogn.

20385

$\mathrm{CR}$

20194

Tibouchina gracilis (Bonpl.) Cogn.

20404

Tibouchina martialis (Cham.) Cogn

20327

Tibouchina ursina (Cham.) Cogn.

20101

Myrtaceae

Campomanesia adamantium (Cambess.) O.Berg

20322

Campomanesia aurea O.Berg

20299

Campomanesia sp.

20312

Myrcia pulchra (O. Berg) Kiaersk.

20224

Psidium grandifolium Mart. ex DC.

20198

Orchidaceae

Cyrtopodium pallidum Rchb.f. \& Warm.*

20290

Cyrtopodium parviflorum Lindl.

20257

Epidendrum secundum Jacq.*

20231

Orobanchaceae

Buchnera ternifolia Kunth*

20335

Esterhazya splendida J.C.Mikan*

CS

20259

Onagraceae

Ludwigia sericea (Cambess.) H. Hara

20220

Ludwigia sp.

CU

20099 
Tabela 1 (continuação)

\begin{tabular}{|c|c|c|}
\hline Família/Espécies & Campo & HUPG \\
\hline \multicolumn{3}{|l|}{ Oxalidaceae } \\
\hline Oxalis hispidula Zucc & $\mathrm{CS}$ & 20263 \\
\hline \multicolumn{3}{|l|}{ Poaceae } \\
\hline Andropogon leucostachyus Kunth* & $\mathrm{CS} / \mathrm{CU} / \mathrm{CR}$ & 20363 \\
\hline Aristida megapotamica Spreng.* & $\mathrm{CS}$ & 20173 \\
\hline Axonopus siccus (Nees) Kuhlm.* & $\mathrm{CR}$ & \\
\hline Brachiaria decumbens Stapf & $\mathrm{CR}$ & 20234 \\
\hline Calamagrostis viridiflavescens (Poir.) Steud.* & $\mathrm{CU}$ & 20280 \\
\hline Chloris gayana Kunth & $\mathrm{CR}$ & 20222 \\
\hline Dichanthelium sabulorum (Lam.) Gould \& C.A. Clark & $\mathrm{CS} / \mathrm{CU}$ & 20285 \\
\hline Digitaria horizontalis Willd. & CS & 20209 \\
\hline Eriochrysis cayennensis P.Beauv.* & $\mathrm{CU}$ & 20341 \\
\hline Panicum glabripes Döll & $\mathrm{CU}$ & 20330 \\
\hline Panicum millegrana Poir.* & CS & 20210 \\
\hline Panicum olyroides Kunth* & $\mathrm{CS}$ & 20317 \\
\hline Panicum peladoense Henrard & $\mathrm{CS}$ & 20343 \\
\hline Panicum sp. 1 & $\mathrm{CU}$ & 20096 \\
\hline Panicum sp. 2 & $\mathrm{CR}$ & \\
\hline Paspalum compressifolium Swallen & $\mathrm{CS}$ & 20261 \\
\hline Paspalum cordatum Hack.* & $\mathrm{CU}$ & 20339 \\
\hline Paspalum erianthum Nees ex Trin.* & $\mathrm{CS}$ & 20292 \\
\hline Paspalum plicatulum Michx.* & $\mathrm{CU}$ & 20265 \\
\hline Paspalum polyphyllum Nees* & $\mathrm{CS}$ & 20350 \\
\hline Paspalum sp. 1 & $\mathrm{CR}$ & 20374 \\
\hline Saccharum villosum Steud. & $\mathrm{CU}$ & 20125 \\
\hline Schizachyrium condensatum (Kunth) Nees* & CS & 20316 \\
\hline Setaria vulpiseta (Lam.) Roem. \& Schult.* & $\mathrm{CR}$ & 20364 \\
\hline Sorghastrum minarum (Nees) Hitchc.* & CS & 20349 \\
\hline Trachypogon spicatus (L.f.) Kuntze* & $\mathrm{CS} / \mathrm{CR}$ & 20348 \\
\hline Trichanthecium cyanescens (Nees ex Trin.) Zuloaga \& Morrone & $\mathrm{CR}$ & 20360 \\
\hline Poaceae 1 & $\mathrm{CS}$ & \\
\hline Poaceae 2 & $\mathrm{CS}$ & \\
\hline Poaceae 3 & $\mathrm{CU}$ & \\
\hline Poaceae 4 & $\mathrm{CS}$ & \\
\hline Poaceae 5 & $\mathrm{CS}$ & \\
\hline Poaceae 6 & $\mathrm{CS}$ & \\
\hline Poaceae 7 & $\mathrm{CS}$ & \\
\hline \multicolumn{3}{|l|}{ Polygalaceae } \\
\hline Polygala hygrophyta Kunth* & $\mathrm{CU}$ & 20236 \\
\hline Polygala longicaulis Kunth* & $\mathrm{CS} / \mathrm{CU}$ & 20161 \\
\hline \multicolumn{3}{|l|}{ Portulacaceae } \\
\hline Portulaca mucronata Link & $\mathrm{CR}$ & \\
\hline
\end{tabular}


Tabela 1 (continuação)

\begin{tabular}{|c|c|c|}
\hline Família/Espécies & Campo & HUPG \\
\hline \multicolumn{3}{|l|}{ Primulaceae } \\
\hline Myrsine umbellata Mart.* & $\mathrm{CR}$ & \\
\hline \multicolumn{3}{|l|}{ Polypodiaceae } \\
\hline Microgramma squamulosa (Kaulf.) de la Sota & $\mathrm{CR}$ & 20386 \\
\hline Pleopeltis lepidopteris (Langsd. \& Fisch.) de la Sota & $\mathrm{CR}$ & 20228 \\
\hline Serpocaulon vacillans (Link) A.R. Sm. & $\mathrm{CR}$ & 20388 \\
\hline \multicolumn{3}{|l|}{ Rhamnaceae } \\
\hline Rhamnus sphaerosperma Sw.* & $\mathrm{CR}$ & 20391 \\
\hline \multicolumn{3}{|l|}{ Rubiaceae } \\
\hline Borreria paranaensis E.L. Cabral \& Bacigalupo & $\mathrm{CS}$ & 20110 \\
\hline Borreria poaya (A. St.-Hil.) DC.* & $\mathrm{CS}$ & 20159 \\
\hline Borreria verticillata (L.) G. Mey. & $\mathrm{CS}$ & 20185 \\
\hline Galianthe chodatiana (Standl.) E.L. Cabral & $\mathrm{CS}$ & 20181 \\
\hline Galianthe elegans E.L. Cabral & CS & 20167 \\
\hline Galium megapotamicum Spreng. & $\mathrm{CS} / \mathrm{CU}$ & 20175 \\
\hline Rubiaceae 1 & CS & 20182 \\
\hline Rubiaceae 2 & $\mathrm{CS}$ & 20329 \\
\hline Rubiaceae 3 & $\mathrm{CS}$ & \\
\hline \multicolumn{3}{|l|}{ Solanaceae } \\
\hline Calibrachoa paranensis (Dusén) Wijsman & $\mathrm{CR}$ & 20225 \\
\hline \multicolumn{3}{|l|}{ Thelypteridaceae } \\
\hline Thelypteris rivularioides (Fée) Abbiatti & $\mathrm{CU}$ & \\
\hline \multicolumn{3}{|l|}{ Turneraceae } \\
\hline Piriqueta suborbicularis (A. St.-Hil. \& Naudin) Arbo & $\mathrm{CS}$ & \\
\hline \multicolumn{3}{|l|}{ Verbenaceae } \\
\hline Lippia hirta Spreng. & $\mathrm{CU}$ & 20244 \\
\hline Lippia lupulina Cham.* & $\mathrm{CS}$ & 20268 \\
\hline Verbena hirta Spreng & $\mathrm{CS}$ & 20323 \\
\hline Verbena sp. 1 & $\mathrm{CS}$ & 20202 \\
\hline \multicolumn{3}{|l|}{ Xyridaceae } \\
\hline Xyris capensis Thunb. & $\mathrm{CU}$ & 20218 \\
\hline Xyris jupicai Rich.* & $\mathrm{CU}$ & 20324 \\
\hline Xyris schizachne Mart.* & $\mathrm{CU}$ & 20340 \\
\hline \multicolumn{3}{|l|}{ Indeterminada } \\
\hline Indeterminada 1 & $\mathrm{CS}$ & \\
\hline Indeterminada 2 & $\mathrm{CS}$ & \\
\hline Indeterminada 3 & $\mathrm{CS}$ & \\
\hline Indeterminada 4 & $\mathrm{CS}$ & \\
\hline Indeterminada 5 & $\mathrm{CU}$ & \\
\hline Indeterminada 6 & $\mathrm{CS}$ & \\
\hline Indeterminada 7 & $\mathrm{CU}$ & \\
\hline Indeterminada 8 & $\mathrm{CU}$ & \\
\hline Indeterminada 9 & $\mathrm{CU}$ & \\
\hline
\end{tabular}


das bandas de confiança das curvas de rarefação indica a riqueza distinta de cada comunidade campestre. A riqueza média por unidade amostral foi de 29,13 para o CS, 14,03 para o CU e 5,46 para o CR. O valor médio de cobertura vegetal no CS foi de $95 \%$, seguidos por $74 \%$ no $\mathrm{CU}$ e $62 \%$ no $\mathrm{CR}$.

Nas fitofisionomias campestres estudadas predominaram espécies herbáceas, especialmente as formas graminosas, intercaladas de indivíduos subarbustivos, sendo que arbustos ou árvores apareceram ocasionalmente. Neste estudo foram amostrados indivíduos de hábito arbóreo em maior número no campo rupestre, sendo representados por Syagrus romanzoffiana, Vitex megapotamica, Cinnamomum sellowianum, Myrcia pulchra, Myrsine umbellata e Rhamnus sphaerosperma. No campo seco foram amostrados Plenckia populnea e Cinnamomum sellowianum e no campo úmido não foram amostrados indivíduos arbóreos.

Das 233 espécies identificadas em nível específico, $36,48 \%$ (85) foram citadas para áreas de Cerrado da região, sendo 45 espécies com ocorrência na área de CS, 31 no CU e 22 no CR. Foram amostradas cinco espécies que constam na Lista Vermelha de Plantas Ameaçadas de Extinção no Estado do Paraná (Paraná 1995): Gochnatia argyrea na categoria "em perigo" e Isostigma speciosum, Mandevilla coccinea,

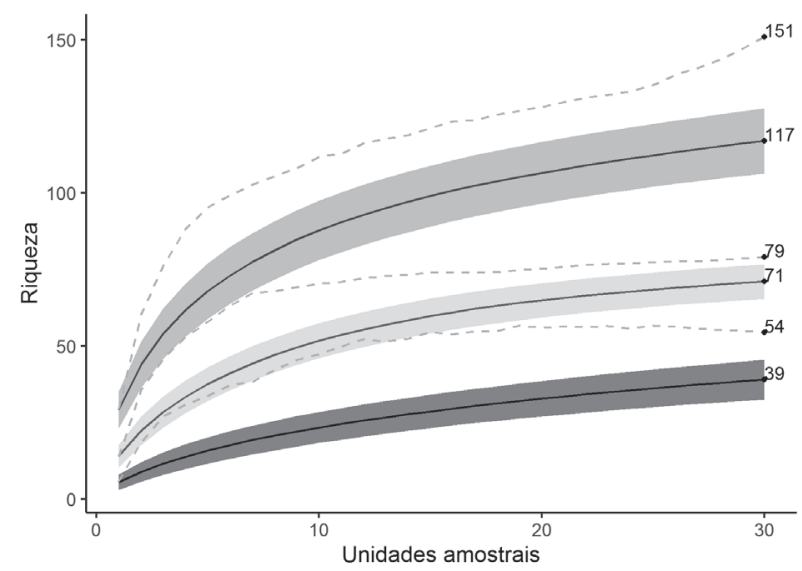

Figura 2. Curvas de rarefação (linhas contínuas), bandas de confiança (área cinza) e estimador de riqueza não paramétrico Chao 2 (linhas pontilhadas) das três fitofisionomias campestres do Parque Estadual de Vila Velha, Ponta Grossa, Paraná. CS: Campo seco, CU: Campo úmido, CR: Campo rupestre. $\square \mathrm{CS}$, $\mathrm{CU}, \square \mathrm{CR}$.

Figure 2. Rarefaction curves (solid lines), confidence bands (gray area) and nonparametric richness estimator Chao 2 (dotted lines) in grassland physiognomies, State Park of Vila Velha, Municipality of Ponta Grossa, Paraná State, Brazil. CS: dry grassland, CU: wet grassland, CR: rock grassland. $\square \mathrm{CS}, \square \mathrm{CU}, \square \mathrm{CR}$.
Gomphrena macrocephala e Cayaponia espelina na categoria "rara".

Estrutura das comunidades - No levantamento fitossociológico das três fitofisionomias foram encontradas 167 espécies (tabela 2), distribuídas em 41 famílias, sendo 97 espécies no CS, 48 no CU e 34 no CR. A espécie que obteve maior VI no campo seco foi Gochnatia argyrea (10,84\%), ocorrendo em 27 das 30 parcelas estudadas. Destacaram-se ainda Calea cuneifolia (9,08\%), Eryngium elegans (8,76\%), Bulbostylis capillaris (8,04\%), Croton antisyphiliticus (7,91\%), Peltodon longipes (7,82\%), Trachypogon spicatus (7,82\%), Stenocephalum megapotamicum (7,23\%), Salvia sp. 1 (5,87\%), Sisyrinchium vaginatum (5,83\%). Os índices dessas espécies somados ao de Gochnatia argyrea corresponderam a $39,5 \%$ do VI total e representaram uma cobertura relativa (CR) de $40,6 \%$.

Para o campo úmido, as espécies de maior importância foram principalmente representantes de Poaceae e Asteraceae, sendo o maior VI para a Rhynchospora sp. 1 (31,0\%), amostrada em 24 das 30 parcelas, seguidas por Paspalum cordatum (19,5\%), Scleria hirtella (18,4\%), Dichanthelium sabulorum (15,9\%), Baccharis crispa (8,9\%), Chromolaena laevigata (7,68\%), Baccharis illinita (6,00\%), Blechnum cordatum (5,25\%), Mikania micrantha e Panicum glabripes (4,8\%). Os índices dessas espécies somaram $61,0 \%$ do VI total e representaram uma cobertura relativa (CR) de $65,0 \%$.

No campo rupestre, as espécies que obtiveram maior VI foram Calea parvifolia (43,5\%), seguida por Axonopus siccus (43,5\%), Polypodium lepidopteris (16,1\%), Sisyrinchium wettsteinii (13,5\%), Symphyopappus cuneatus (8,9\%), Calibrachoa paranensis (8,6\%), Panicum parvifolium (7,05\%), Praxelis sanctopaulensis (6,7\%), Epidendrum secundum (5,7\%) e Trachypogon spicatus (5,2\%). Os índices dessas espécies detêm 78,9\% do VI total e representaram uma cobertura relativa (CR) de $82,2 \%$.

A análise de cluster agrupou homogeneamente as parcelas pertencentes a cada formação campestre (figura 3), o desempenho do agrupamento foi quase perfeito por ter uma correlação cofenética de 0,97 . O teste MRPP apontou que as composições florísticas das três formações campestres são significativamente distintas entre si ( $\delta$ observado: $0,57, \delta$ estimado: 0,85 $\mathrm{P}<0,001)$, mas com certa heterogeneidade entre os elementos que compõem cada grupo (A: 0,33 ), evidenciando a diversidade florística de cada parcela. 
Tabela 2. Relação das espécies amostradas no levantamento fitossociológico nas fitofisionomias campestres do Parque Estadual de Vila Velha, Ponta Grossa, Paraná. CS: Campo seco, CU: Campo úmido, CR: Campo rupestre, Pi: nº de amostras que a espécie ocorre, FR: frequência relativa, CR: cobertura relativa, VI: valor de importância.

Table 2. Phytosociological parameters of the species sampled in grassland physiognomies, State Park of Vila Velha, Municipality of Ponta Grossa, Paraná State, Brazil. CS: dry grassland, CU: wet grassland, CR: rocky grassland, Pi: number of samples where the species occurs, FR: relative frequency, CR: relative coverage, VI: importance value.

\begin{tabular}{|c|c|c|c|c|c|c|c|c|c|c|c|c|}
\hline Espécies & & $\mathrm{Pi}$ & & & FR & & & $\mathrm{Cr}$ & & & VI & \\
\hline & $\mathrm{CS}$ & $\mathrm{CU}$ & $\mathrm{CR}$ & $\mathrm{CS}$ & $\mathrm{CU}$ & $\mathrm{CR}$ & $\mathrm{CS}$ & $\mathrm{CU}$ & $\mathrm{CR}$ & $\mathrm{CS}$ & $\mathrm{CU}$ & $\mathrm{CR}$ \\
\hline Achyrocline satureioides & & 2 & 1 & & 0,88 & 0,71 & & 0,62 & 0,4 & & 1,5 & 1,11 \\
\hline Acisanthera quadrata & & 1 & & & 0,44 & & & 0,31 & & & 0,75 & \\
\hline Aechmea distichantha & & & 1 & & & 0,71 & & & 0,4 & & & 1,11 \\
\hline Aldama kunthiana & 14 & & & 2,53 & & & 2,31 & & & 4,83 & & \\
\hline Allagoptera campestris & 4 & & 2 & 0,72 & & 1,43 & 0,81 & & 1,19 & 1,54 & & 2,61 \\
\hline Alternanthera brasiliana & 1 & & & 0,18 & & & 0,14 & & & 0,32 & & \\
\hline Andropogon leucostachyus & & & 2 & & & 1,43 & & & 0,79 & & & 2,22 \\
\hline Aspicarpa pulchella & 2 & & & 0,36 & & & 0,27 & & & 0,63 & & \\
\hline Asteraceae 6 & & 5 & & & 2,2 & & & 1,86 & & & 4,06 & \\
\hline Asteraceae 7 & & 1 & & & 0,44 & & & 0,31 & & & 0,75 & \\
\hline Asteraceae 8 & 1 & & & 0,18 & & & 0,14 & & & 0,32 & & \\
\hline Austroeupatorium inulaefolium & & 3 & & & 1,32 & & & 1,55 & & & 2,87 & \\
\hline Axonopus siccus & & & 26 & & & 18,57 & & & 24,9 & & & 43,47 \\
\hline Baccharis crispa & & 11 & & & 4,85 & & & 4,02 & & & 8,87 & \\
\hline Baccharis illinita & & 8 & & & 3,52 & & & 2,48 & & & 6 & \\
\hline Baccharis myricifolia & 1 & & & 0,18 & & & 0,14 & & & 0,32 & & \\
\hline Blechnum cordatum & & 7 & & & 3,08 & & & 2,17 & & & 5,25 & \\
\hline Borreria paranaensis & 11 & & & 1,99 & & & 1,49 & & & 3,48 & & \\
\hline Borreria poaya & 10 & & & 1,81 & & & 1,36 & & & 3,16 & & \\
\hline Borreria verticillata & 3 & & & 0,54 & & & 0,41 & & & 0,95 & & \\
\hline Brachiaria decumbens & & & 1 & & & 0,71 & & & 0,4 & & & 1,11 \\
\hline Bulbostylis juncoides & 1 & & & 0,18 & & & 0,14 & & & 0,32 & & \\
\hline Bulbostyllis capillaris & 22 & & 1 & 3,97 & & 0,71 & 4,07 & & 0,4 & 8,04 & & 1,11 \\
\hline Bulbostyllis sp. 1 & & 3 & & & 1,32 & & & 0,93 & & & 2,25 & \\
\hline Butia microspadix & 5 & & & 0,9 & & & 0,81 & & & 1,72 & & \\
\hline Byrsonima brachybotrya & 5 & & & 0,9 & & & 0,95 & & & 1,85 & & \\
\hline Byrsonima guilleminiana & 2 & & & 0,36 & & & 0,54 & & & 0,9 & & \\
\hline Byttneria hatschbachii & 2 & & & 0,36 & & & 0,27 & & & 0,63 & & \\
\hline Calea cuneifolia & 24 & & & 4,33 & & & 4,75 & & & 9,08 & & \\
\hline Calea cymosa & 7 & & & 1,26 & & & 1,36 & & & 2,62 & & \\
\hline Calea parvifolia & & & 25 & & & 17,86 & & & 25,69 & & & 43,55 \\
\hline Calibrachoa paranensis & & & 7 & & & 5 & & & 3,56 & & & 8,56 \\
\hline Calydorea campestris & & 4 & & & 1,76 & & & 1,24 & & & 3 & \\
\hline Campomanesia aurea & 6 & & & 1,08 & & & 1,49 & & & 2,58 & & \\
\hline Campomanesia sp. & 1 & & & 0,18 & & & 0,27 & & & 0,45 & & \\
\hline Campovassouria cruciata & & 6 & & & 2,64 & & & 2,17 & & & 4,81 & \\
\hline
\end{tabular}


Tabela 2 (continuação)

\begin{tabular}{|c|c|c|c|c|c|c|c|c|c|c|}
\hline Espécies & & $\mathrm{Pi}$ & & FR & & & $\mathrm{Cr}$ & & VI & \\
\hline Chamaecrista puntacta & 9 & & 1,62 & & & 2,85 & & 4,47 & & \\
\hline Chamaecrista ramosa & 2 & & 0,36 & & & 0,27 & & 0,63 & & \\
\hline Chamaecrista vestita & 2 & & 0,36 & & & 0,27 & & 0,63 & & \\
\hline Chloris gayana & & & 1 & & 0,71 & & & 0,79 & & 1,5 \\
\hline Chromolaena congesta & & & 1 & & 0,71 & & & 0,4 & & 1,11 \\
\hline Chromolaena laevigata & 1 & 9 & 0,18 & 3,96 & & 0,14 & 3,72 & 0,32 & 7,68 & \\
\hline Chromolaena palmaris & 3 & & 0,54 & & & 0,41 & & 0,95 & & \\
\hline Chrysolaena platensis & 8 & & 1,44 & & & 1,36 & & 2,8 & & \\
\hline Chrysolaena simplex & 1 & & 0,18 & & & 0,14 & & 0,32 & & \\
\hline Cinnamomum sellowianum & & & 1 & & 0,71 & & & 0,4 & & 1,11 \\
\hline Commelina villosa & & & 2 & & 1,43 & & & 1,19 & & 2,61 \\
\hline Croton antisyphiliticus & 22 & & 3,97 & & & 3,93 & & 7,91 & & \\
\hline Croton heterodoxus & 4 & & 0,72 & & & 0,54 & & 1,26 & & \\
\hline Croton solanaceus & 2 & & 0,36 & & & 0,27 & & 0,63 & & \\
\hline Cuphea linifolia & 3 & & 0,54 & & & 0,41 & & 0,95 & & \\
\hline Cyperaceae sp. 1 & & 4 & & 1,76 & & & 1,24 & & 3 & \\
\hline Cyperaceae sp. 2 & & 1 & & 0,44 & & & 0,62 & & 1,06 & \\
\hline Cyperus agreggatus & & & 1 & & 0,71 & & & 0,4 & & 1,11 \\
\hline Cyperus rotundus & & 1 & & 0,44 & & & 0,31 & & 0,75 & \\
\hline Desmodium barbatum & & 2 & & 0,88 & & & 0,62 & & 1,5 & \\
\hline Dichanthelium sabulorum & & 20 & & 8,81 & & & 7,12 & & 15,93 & \\
\hline Epidendrum secundum & & & 3 & & 2,14 & & & 3,56 & & 5,7 \\
\hline Eriosema glabra & 11 & & 1,99 & & & 2,85 & & 4,83 & & \\
\hline Eriosema heterophyllum & 1 & & 0,18 & & & 0,14 & & 0,32 & & \\
\hline Eryngium ebracteatum & & 3 & & 1,32 & & & 0,93 & & 2,25 & \\
\hline Eryngium elegans & 23 & & 4,15 & & & 4,61 & & 8,76 & & \\
\hline Eryngium horridum & 1 & & 0,18 & & & 0,14 & & 0,32 & & \\
\hline Eryngium sanguisorba & 3 & & 0,54 & & & 0,41 & & 0,95 & & \\
\hline Erythroxylum microphyllum & 10 & & 1,81 & & & 1,49 & & 3,3 & & \\
\hline Eupatorium sp. 1 & 1 & & 0,18 & & & 0,14 & & 0,32 & & \\
\hline Eupatorium sp. 2 & 8 & & 1,44 & & & 1,36 & & 2,8 & & \\
\hline Evolvulus alsinoides & 4 & & 0,72 & & & 0,54 & & 1,26 & & \\
\hline Galianthe elegans & 4 & & 0,72 & & & 0,68 & & 1,4 & & \\
\hline Galium megapotamicum & 3 & 1 & 0,54 & 0,44 & & 0,41 & 0,31 & 0,95 & 0,75 & \\
\hline Gamochaeta purpurea & & 3 & & 1,32 & & & 1,24 & & 2,56 & \\
\hline Gochnatia argyrea & 27 & & 4,87 & & & 5,97 & & 10,84 & & \\
\hline Gyptis lanigera & 3 & & 0,54 & & & 0,54 & & 1,08 & & \\
\hline Heterocondylus pumilus & 5 & & 0,9 & & & 0,68 & & 1,58 & & \\
\hline Hypericum cordatum & 1 & & 0,18 & & & 0,14 & & 0,32 & & \\
\hline Ilex chamaedryfolia & 3 & & 0,54 & & & 0,54 & & 1,08 & & \\
\hline Isostigma speciosum & 3 & & 0,54 & & & 0,68 & & 1,22 & & \\
\hline Krapovickasia macrodon & 1 & & 0,18 & & & 0,14 & & 0,32 & & \\
\hline
\end{tabular}


Tabela 2 (continuação)

\begin{tabular}{|c|c|c|c|c|c|c|c|c|c|c|}
\hline Espécies & & $\mathrm{Pi}$ & & & FR & & $\mathrm{Cr}$ & & VI & \\
\hline Lavoisiera pulchella & & 5 & & & 2,2 & & 1,86 & & 4,06 & \\
\hline Leandra erostrata & 8 & & & 1,44 & & 1,22 & & 2,67 & & \\
\hline Lessingianthus grandiflorus & 2 & & & 0,36 & & 0,27 & & 0,63 & & \\
\hline Lessingianthus rubricaulis & 5 & & & 0,9 & & 0,68 & & 1,58 & & \\
\hline Lippia lupulina & 3 & & & 0,54 & & 0,41 & & 0,95 & & \\
\hline Lobelia camporum & 3 & & & 0,54 & & 0,41 & & 0,95 & & \\
\hline Lucilia lycopodioides & 13 & & & 2,35 & & 1,9 & & 4,25 & & \\
\hline Mandevilla pohliana & 1 & & & 0,18 & & 0,14 & & 0,32 & & \\
\hline Mikania micrantha & & 6 & & & 2,64 & & 2,17 & & 4,81 & \\
\hline Mikania oblongifolia & 1 & & & 0,18 & & 0,14 & & 0,32 & & \\
\hline Moritzia dusenii & 4 & & & 0,72 & & 0,54 & & 1,26 & & \\
\hline Myrcia pulchra & & & 1 & & 0,71 & & 1,19 & & & 1,9 \\
\hline Oxypetalum sublanatum & & & 3 & & 2,14 & & 1,19 & & & 3,33 \\
\hline Paepalanthus caldensis & & 4 & & & 1,76 & & 1,24 & & 3 & \\
\hline Panicum glabripes & & 6 & & & 2,64 & & 2,17 & & 4,81 & \\
\hline Panicum millegrana & 1 & & & 0,18 & & 0,14 & & 0,32 & & \\
\hline Panicum parvifolium & & & 6 & & 4,29 & & 2,77 & & & 7,05 \\
\hline Panicum sabulorum & 2 & & & 0,36 & & 0,27 & & 0,63 & & \\
\hline Panicum sp.1 & & 1 & & & 0,44 & & 0,62 & & 1,06 & \\
\hline Paspalum cordatum & & 19 & & & 8,37 & & 11,15 & & 19,52 & \\
\hline Paspalum erianthum & 13 & & & 2,35 & & 2,58 & & 4,92 & & \\
\hline Paspalum polyphyllum & & & 2 & & 1,43 & & 1,19 & & & 2,61 \\
\hline Paspalum sp.2 & 3 & & & 0,54 & & 0,41 & & 0,95 & & \\
\hline Pavonia speciosa & 10 & & & 1,81 & & 2,44 & & 4,25 & & \\
\hline Peltodon longipes & 20 & & & 3,61 & & 4,21 & & 7,82 & & \\
\hline Pfaffia gnaphaloides & & 3 & & & 1,32 & & 1,24 & & 2,56 & \\
\hline Piriqueta suborbicularis & 2 & & & 0,36 & & 0,27 & & 0,63 & & \\
\hline Pleopeltis lepidopteris & & & 12 & & 8,57 & & 7,51 & & & 16,08 \\
\hline Poaceae 1 & 10 & & & 1,81 & & 2,17 & & 3,98 & & \\
\hline Poaceae 2 & 1 & & & 0,18 & & 0,14 & & 0,32 & & \\
\hline Poaceae 3 & & 3 & & & 1,32 & & 1,55 & & 2,87 & \\
\hline Poaceae 4 & 1 & & & 0,18 & & 0,27 & & 0,45 & & \\
\hline Poaceae 5 & 2 & & & 0,36 & & 0,27 & & 0,63 & & \\
\hline Poaceae 6 & 13 & & & 2,35 & & 3,12 & & 5,47 & & \\
\hline Polygala longicaulis & 3 & 1 & & 0,54 & 0,44 & 0,41 & 0,31 & 0,95 & 0,75 & \\
\hline Portulaca mucronata & & & 1 & & 0,71 & & 0,4 & & & 1,11 \\
\hline Praxelis sanctopaulensis & & & 6 & & 4,29 & & 2,37 & & & 6,66 \\
\hline Psidium grandifolium & 2 & & & 0,36 & & 0,27 & & 0,63 & & \\
\hline Pteridium arachnoideum & 1 & & & 0,18 & & 0,27 & & 0,45 & & \\
\hline Pterocaulon angustifolium & 6 & & & 1,08 & & 0,81 & & 1,9 & & \\
\hline Pterocaulon balansae & & 3 & & & 1,32 & & 0,93 & & 2,25 & \\
\hline Pycreus polystachyos & & 1 & & & 0,44 & & 0,31 & & 0,75 & \\
\hline
\end{tabular}


Tabela 2 (continuação)

\begin{tabular}{|c|c|c|c|c|c|c|c|c|c|c|c|c|}
\hline \multirow{2}{*}{$\frac{\text { Espécies }}{\text { Rhamnus sphaerosperma }}$} & \multicolumn{3}{|c|}{$\mathrm{Pi}$} & \multicolumn{3}{|c|}{ FR } & \multicolumn{3}{|c|}{$\mathrm{Cr}$} & \multicolumn{3}{|c|}{ VI } \\
\hline & & & 1 & & & 0,71 & & & 0,4 & & & 1,11 \\
\hline Rhynchosia corydifolia & 3 & & & 0,54 & & & 0,54 & & & 1,08 & & \\
\hline Rhynchospora albicans & & 3 & & & 1,32 & & & 1,55 & & & 2,87 & \\
\hline Rhynchospora globosa & & 1 & & & 0,44 & & & 0,31 & & & 0,75 & \\
\hline Rhynchospora rugosa & & 4 & & & 1,76 & & & 0,62 & & & 2,38 & \\
\hline Rhynchospora sp. 1 & & 24 & & & 10,57 & & & 20,43 & & & 31,01 & \\
\hline Saccharum villosum & & 3 & & & 1,32 & & & 1,24 & & & 2,56 & \\
\hline Salvia aliciae & 5 & & & 0,9 & & & 0,81 & & & 1,72 & & \\
\hline Salvia sp. 1 & 16 & & & 2,89 & & & 2,99 & & & 5,87 & & \\
\hline Scleria hirtella & & 20 & & & 8,81 & & & 9,6 & & & 18,41 & \\
\hline Serpocaulon vaccilans & & & 1 & & & 0,71 & & & 0,4 & & & 1,11 \\
\hline Sida sp. & 1 & & & 0,18 & & & 0,14 & & & 0,32 & & \\
\hline Sisyrinchium restioides & 12 & & & 2,17 & & & 2,04 & & & 4,2 & & \\
\hline Sisyrinchium vaginatum & 18 & 4 & & 3,25 & 1,76 & & 2,58 & 1,55 & & 5,83 & 3,31 & \\
\hline Sisyrinchium wettsteinii & & & 10 & & & 7,14 & & & 6,32 & & & 13,47 \\
\hline Stenocephalum megapotamicum & 22 & & & 3,97 & & & 3,26 & & & 7,23 & & \\
\hline Stevia leptophylla & & & 3 & & & 2,14 & & & 1,98 & & & 4,12 \\
\hline Stylosanthes hippocampoides & 1 & & & 0,18 & & & 0,14 & & & 0,32 & & \\
\hline Stylosanthes montevidensis & 1 & & & 0,18 & & & 0,14 & & & 0,32 & & \\
\hline Symphyopappus cuneatus & & & 8 & & & 5,71 & & & 3,16 & & & 8,88 \\
\hline Thelypteris rivularioides & & 4 & & & 1,76 & & & 1,24 & & & 3 & \\
\hline Tibouchina debilis & 3 & & & 0,54 & & & 0,41 & & & 0,95 & & \\
\hline Tibouchina gracilis & & 6 & & & 2,64 & & & 2,17 & & & 4,81 & \\
\hline Tibouchina martialis & 2 & & & 0,36 & & & 0,41 & & & 0,77 & & \\
\hline Tibouchina ursina & & 2 & & & 0,88 & & & 0,62 & & & 1,5 & \\
\hline Tillandsia stricta & & & 1 & & & 0,71 & & & 0,4 & & & 1,11 \\
\hline Trachypogon spicatus & 20 & & 4 & 3,61 & & 2,86 & 4,21 & & 2,37 & 7,82 & & 5,23 \\
\hline Trichanthecium cyanescens & & & 1 & & & 0,71 & & & 0,4 & & & 1,11 \\
\hline Trichocline speciosa & 3 & & & 0,54 & & & 0,41 & & & 0,95 & & \\
\hline Stylosanthes guianensis & 3 & & & 0,54 & & & 0,41 & & & 0,95 & & \\
\hline Verbesina sordescens & 1 & & & 0,18 & & & 0,27 & & & 0,45 & & \\
\hline Vernonanthura crassa & & & 2 & & & 1,43 & & & 1,58 & & & 3,01 \\
\hline Vernonanthura nudiflora & 1 & & & 0,18 & & & 0,14 & & & 0,32 & & \\
\hline Vernonanthura sp. 1 & 1 & & & 0,18 & & & 0,14 & & & 0,32 & & \\
\hline Vitex megapotamica & & & 1 & & & 0,71 & & & 0,4 & & & 1,11 \\
\hline Vittetia orbiculata & 9 & & & 1,62 & & & 1,76 & & & 3,39 & & \\
\hline Vriesea friburgensis & & & 2 & & & 1,43 & & & 1,58 & & & 3,01 \\
\hline Waltheria communis & 4 & & & 0,72 & & & 0,54 & & & 1,26 & & \\
\hline Xyris jupicai & & 1 & & & 0,44 & & & 0,31 & & & 0,75 & \\
\hline Xyris schizachne & & 1 & & & 0,44 & & & 0,31 & & & 0,75 & \\
\hline Zornia criptantha & 3 & & & 0,54 & & & 0,41 & & & 0,95 & & \\
\hline Indeterminada 1 & 1 & & & 0,18 & & & 0,27 & & & 0,45 & & \\
\hline Indeterminada 2 & 1 & & & 0,18 & & & 0,27 & & & 0,45 & & \\
\hline Indeterminada 3 & 1 & & & 0,18 & & & 0,14 & & & 0,32 & & \\
\hline Indeterminada 4 & 1 & & & 0,18 & & & 0,14 & & & 0,32 & & \\
\hline Indeterminada 5 & & 2 & & & 0,88 & & & 0,62 & & & 1,5 & \\
\hline Indeterminada 7 & & 1 & & & 0,44 & & & 0,31 & & & 0,75 & \\
\hline
\end{tabular}


A análise de ordenação mostrou a singularidade florística de cada comunidade devido à distribuição das unidades amostrais nos eixos de ordenação (figura 4), o valor de estresse foi de 0,06 , valor quase excelente, o que indica um bom ajuste entre a matriz de distância original e o posicionamento dos elementos nos dois eixos de ordenação. O teste de Mantel acusou que os eixos são significativos (Mantel r: 0,90 $\mathrm{P}<0,001$ ). $\mathrm{O}$ primeiro eixo de ordenação pode ser interpretado

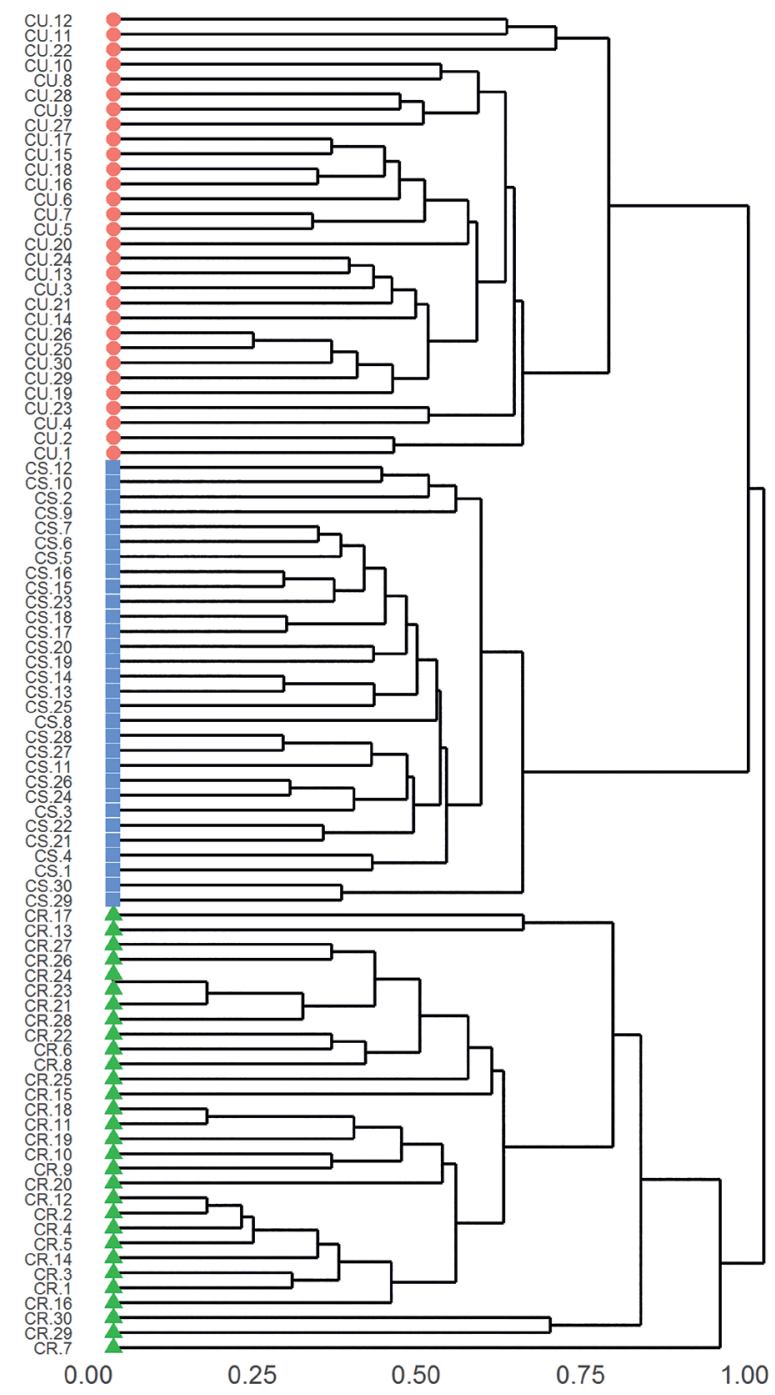

Figura 3. Dendrograma da análise de agrupamento UPGMA com índice de similaridade de Sorensen para as espécies amostradas em três fitofisionomias campestres do Parque Estadual de Vila Velha, Ponta Grossa, Paraná. CS: Campo seco; CU: Campo úmido; CR: Campo rupestre. $\mathrm{CU}, \square \mathrm{CS}, \triangle \mathrm{CR}$.

Figure 3. Dendrogram based on cluster analysis (UPGMA) using Sorensen Index for the species sampled in grasslands of Vila Velha State Park, Ponta Grossa, Paraná State, Brazil. CS: dry grassland; CU: wet grassland; CR: rocky grassland. $\mathrm{CU}$, $\square \mathrm{CS}, \triangle \mathrm{CR}$. como um gradiente de saturação hídrica, já que, de esquerda à direita foram ordenadas as unidades amostrais do CU, seguido do CS e finalmente o CR. E o segundo eixo indica a similaridade florística entre as áreas.

\section{Discussão}

Apesar da grande variação na riqueza florística nos campos do Sul do Brasil (Boldrini \& Miotto 1987, Kozera et al. 2009, Dalazoana \& Moro 2011, Moro et al. 2012), os trabalhos na região dos Campos Gerais do Paraná têm registrado número de espécies superior (Ferreira \& Setubal 2009, Boldrini et al. 2008, Setubal \& Boldrini 2012). Neste estudo a escolha dos métodos contribuiu para alcançar alta riqueza, já que o levantamento adicional de até $50 \mathrm{~m}$ de distância das unidades amostrais representou quase $40 \%$ do total de espécies amostradas. Fatores como fogo (Overbeck et al. 2005, Overbeck et al. 2009) e localização setentrional dos campos do Sul do Brasil, com influência da transição entre climas Tropical e Subtropical (Maack 2012), somados a presença de elementos disjuntos de Cerrado (Ritter et al. 2010), devem estar contribuindo para a alta riqueza florística encontrada nos campos do PEVV.

Asteraceae e Poaceae foram as famílias mais representativas nas três fitofisionomias, corroborando com os resultados encontrados na região dos Campos Gerais por Hatschbach \& Moreira Filho (1972), Cervi et al. (2003), Carmo (2006), Cervi et al. (2007), Dalazoana et al. (2007), Nanuncio \& Moro (2008), Kozera et al. (2009), Dalazoana \& Moro (2011), Moro et al. (2012). Tais famílias são predominantes nas comunidades campestres do Sul do Brasil, conforme também observado por Boldrini \& Miotto (1987), Longhi-Wagner (2003), Boldrini (2009) e Selusniaki (2013). Vale destacar que estudos realizados por Cervi et al. (2007) no PEVV apontam que 63\% das espécies de gramíneas são compartilhadas com áreas de Cerrado.

Cyperaceae também foi uma das famílias mais representativas no $\mathrm{CU}$, devido a sua adaptação a áreas hidromórficas (Souza \& Lorenzi 2008), como já relatados em outros estudos (Carmo 2006, Kozera et al. 2009, Andrade et al. 2011). Já no CR, Bromeliaceae foi uma das famílias mais representativas, com ocorrência de espécies típicas (Carmo et al. 2014), como Aechmea distichantha, Tillandsia stricta e Tillandsia crocata.

Algumas espécies amostradas, como Allagoptera campestris e Plenckia populnea, são típicas das 
savanas brasileiras, relictos de uma vegetação que dominou o Estado do Paraná em épocas pretéritas (Maack 1981). Estudos realizados por Ritter et al. (2010) apontam que, floristicamente, a vegetação do PEVV exibe atributos qualitativos suficientes para ser considerada uma fitocenose de Cerrado, corroborando com os estudos realizados por Cervi et al. (2007).

$\mathrm{Na}$ vegetação dos Campos Gerais são encontradas várias espécies listadas como ameaçadas de extinção para o Estado do Paraná (Ziller \& Hatschbach 1996, Kozera et al. 2009, Carmo et al. 2012), o que ressalta a importância desta região e a necessidade de ampliação de áreas protegidas para conservação da biodiversidade (Moro \& Carmo 2007). Neste estudo, as espécies ameaçadas representaram 1,73\%, podendo estar subestimadas, já que $3,18 \%$ das espécies amostradas não foram, até o momento, identificadas por estarem no estado vegetativo e/ou de plântula.

Entre as três fisionomias campestres, a menor riqueza de espécies foi registrada no $\mathrm{CR}$, resultado esperado já que os solos são incipientes, restritos às fraturas das rochas, diminuindo a área para a colonização das espécies vegetais (média de cobertura vegetal de 62\%). Além disso, o substrato presente nas fendas apresenta grande variação de temperatura (Conceição et al. 2007, Oliveira \& Godoy 2007), dificultando ainda mais o estabelecimento de um

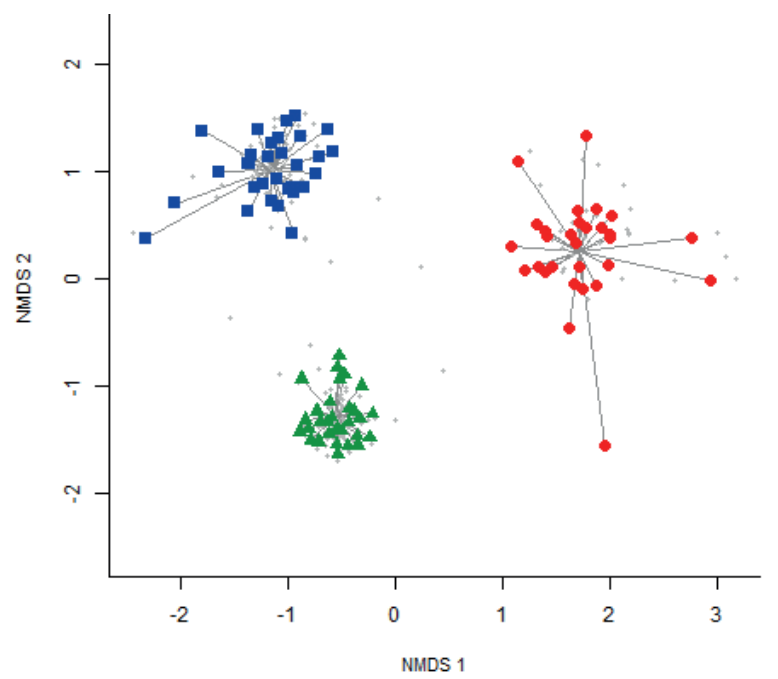

Figura 4. Diagrama de ordenação da análise de NMDS das três fitofisionomias campestres amostradas no Parque Estadual de Vila Velha, Ponta Grossa, Paraná. CS: Campo seco; CU: Campo úmido; CR: Campo rupestre. $\square \mathrm{CU}, \triangle \mathrm{CS}, \mathrm{CR}$.

Figure 4. Ordination diagram of the NMDS analysis in grassland physiognomies, State Park of Vila Velha, Municipality of Ponta Grossa, Paraná State, Brazil. CS: dry grassland; CU: wet grassland; $\mathrm{CR}$ : rocky grassland. $\square \mathrm{CU}, \triangle \mathrm{CS}, \odot \mathrm{CR}$. número maior de espécies, por ter que se adaptar ao microclima seletivo (Cervi et al. 2007).

Vale destacar que na área de CR foi amostrado maior número de indivíduos arbóreos, e isso pode estar associado à ausência de registros de queimadas, contribuindo para o recrutamento de árvores e arbustos, o que corrobora o fato do fogo ser apontado como uma das causas da diminuição do avanço da floresta sobre os campos (Frost \& Robertson 1987, Agee 1993, Medina \& Huber 1994). Se a supressão do fogo for mantida nessas áreas, é possível que os campos em unidades de conservação reduzam a sua expressividade com a expansão florestal, a exemplo do que ocorreu na pós-glaciação em grande parte do Sul do Brasil (Müller et al. 2012, Fidelis et al. 2007, Behling et al. 2009).

Embora a vegetação campestre das diferentes fitofisionomias do PEVV apresente uma aparente cobertura homogênea, neste estudo foi possível constatar que as fitofisionomias são significativamente distintas entre si, conforme observado na análise de agrupamento, o que indica uma alta especificidade da vegetação encontrada nessas áreas. A distinção entre a composição florística dessas fitofisionomias se deve a diversos fatores, tais como: regime hídrico do solo e seu grau de saturação, relacionados à posição que ocupam na encosta, bem como forma e declividade da rampa, altitude, espessura do solo, textura e forma de contato com a rocha, entre outros. A combinação desses elementos pode resultar em escoamentos hídricos distintos, os quais são determinantes para a presença de algumas espécies e para a formação de mosaicos tipológicos (Curcio et al. 2004), que refletem diretamente na densidade, composição e estrutura do banco de sementes, sendo o regime hídrico do solo, um dos principais fatores ambientais determinante nos padrões de distribuição espacial nas comunidades vegetais (Cabido et al.1987).

O menor valor de similaridade encontrado foi entre o CU e o CR, também observado por Dalazoana et al. 2007, no mesmo local deste estudo, confirmando a relevante diferenciação entre os ambientes. Ainda, estes mesmos autores observaram que os campos rupestres mostraram-se associados a grupos de campos secos. Porém tais resultados não foram observados neste estudo, embora as similaridades tenham sido baixas entre as áreas. O CS se mostrou mais associado às áreas de $\mathrm{CU}$, contudo, estatisticamente estas fisionomias foram consideradas distintas.

De qualquer maneira, nas três áreas estudadas foram amostradas somente três espécies comuns, 
evidenciando que nas fitofisionomias podem ocorrer espécies com grande plasticidade, podendo se estabelecer em condições hídricas e edáficas muito distintas, conforme também observado por Carmo (2006), em outra Unidade Conservação na região dos Campos Gerais.

A espécie de maior VI no campo seco, Gochnatia argyrea, tem sido amostrada com menor expressividade (Carmo et al. 2012) ou está ausente nas listas de espécies de estudos realizados na região (Dalazoana et al. 2007, Kozera et al. 2009, Dalazoana \& Moro 2011, Ferreira \& Maranho 2011, Moro et al. 2012), sendo citada na Lista Vermelha de Plantas Ameaçadas de Extinção no Estado do Paraná na categoria em perigo (Paraná 1995). A abundância de tal espécie pode estar relacionada às frequentes queimadas na área de estudo, as quais podem influenciar diretamente a composição (Coutinho 1981, Whelan 1997, Carmo et al. 2007, Miranda et al. 2009), bem como o banco de sementes do solo (Heringer \& Jacques 2001, Andrade et al. 2002), pois apresenta oscilações nas diferentes estações, refletindo diretamente na densidade e frequência dos indivíduos e riqueza de espécies (Chaghtai et al. 1985, Longhi et al. 2005).

Estudos pontuais realizados no PEVV (Ferreira \& Maranho 2011, Oliveira \& Maranho 2011) indicam que o fogo possibilita uma redistribuição da comunidade, promovendo redução das espécies adultas e dominantes, possibilitando o aumento no número de espécies herbáceas. Estes dados reforçam ainda mais o papel do fogo para a manutenção da diversidade dos campos (Overbeck et al. 2009).

A manutenção e conservação das fitofisionomias campestres do PEVV dependem da intervenção humana, dado ao avanço natural das florestas substituindo os ambientes campestres. Um plano de manejo com fogo controlado nas áreas campestres do parque pode ser uma alternativa para manter artificialmente essas fitofisionomias, e evitar uma redução natural da biodiversidade e extinção de espécies.

Portanto, trabalhos que enfoquem o comportamento da vegetação sob diferentes condições ambientais devem ser ampliados, para possibilitar melhor compreensão dos processos relacionados à dinâmica da vegetação, levando em conta as características ambientais de cada localidade.

\section{Agradecimentos}

À CAPES, pela Bolsa concedida à primeira Autora, aos funcionários do Museu Botânico
Municipal - MBM (Curitiba/Paraná), em especial ao Biólogo Osmar dos Santos Ribas, pela preciosa ajuda com a identificação das exsicatas, à professora Rosangela Capuano Tardivo, curadora do Herbário da Universidade Estadual de Ponta Grossa - HUPG/ UEPG, por permitir o uso das instalações e à Pró Reitoria de Extensão e Assuntos Culturais - PROEX/ UEPG pelo apoio logístico.

\section{Literatura citada}

APG III (The Angiosperm Phylogeny Group). 2009. An update of the Angiosperm Phylogeny Group classification for the orders and families of flowering plants: APG III. Botanical Journal of the Linnean Society 161: 105-121.

Agee, J.K. 1993. Fire Ecology of Pacific Northwest Forests. New York, Island Press.

Andrade, L.A.Z., Neto, W.N. \& Miranda, H.S. 2002. Effects of fire on the soil seed bank in a cerrado sensu stricto in central Brazil. In: D.X. Viegas (ed.). Forest fire research and wildland fire safety: Proceedings of IV International Conference on Forest Fire Research. Coimbra, Portugal. Millpress, Roterdam. 217: 18-23.

Andrade, B.O., Kozera, C., Curcio, G.R. \& Galvão, F. 2011. Vascular grassland plants of Tibagi River Spring, Ponta Grossa, Brazil. Check List. Journal of species lists and distribution 7: 257-262. Disponível em http://www. checklist.org.br/getpdf?SL073-10 (acesso em 20-XII-2013).

Behling, H. \& Pillar, V.D.P. 2007. Late Quaternary vegetation, biodiversity and fire dynamics on the southern Brazilian highland and their implication for conservation and management of modern Araucaria forest and grassland ecosystems. Philosophical Transactions Royal Society 362: 243-251.

Behling, H., Jeske-Pieruschka, J., Schüler, L. \& Pillar, V.D.P. 2009. Dinâmica dos campos no sul do Brasil durante o Quaternário Tardio. In: V.P. Pillar, S.C. Müller, Z.M.S. Castilhos, \& A.V.A. Jacques (eds.). Campos Sulinos - conservação e uso sustentável da biodiversidade. Brasília: MMA. pp. 13-25.

Boldrini, I.I. 2009. A flora dos Campos do Rio Grande do Sul. In: V.D.P. Pillar, S.C. Müller, Z.M.S. Castilhos \& A.V.A Jacques (eds.). Campos Sulinos: conservação e uso sustentável da biodiversidade. Ministério do Meio Ambiente, Brasília, pp. 63-77.

Boldrini, I.I. \& Miotto, S.T.S. 1987. Levantamento Fitossociológico de um Campo Limpo da Estação Experimental Agronômica, UFRGS, Guaíba, RS - $1^{\text {a }}$ Etapa. Acta Botânica Brasílica, São Paulo 1: 49-56.

Boldrini, I.I., Trevisan, R. \& Schneider, A.A. 2008. Estudo florístico e fitossociológico de uma área às margens da lagoa do Armazém, Osório, Rio Grande do Sul, Brasil. Revista Brasileira de Biociências, Porto Alegre, 6: 355-367. 
Cabido, M., Breimer, R. \& Veja, G. 1987. Plant communities and associated soil types in a high plateau of the Córdoba mountains, Central Argentina. Mountain Research and Development 7: 25-42.

Caramari, P.H. 2004. Climatologia. In: Plano de manejo Parque Estadual de Vila Velha. Curitiba: IAP - Instituto Ambiental do Paraná, SEMA - Secretaria do Estado e do Meio Ambiente e Recursos Hídricos. pp. 136-174.

Carmo, M.R.B. 2006. Caracterização fitofisionômica do Parque Estadual do Guartelá, Município de Tibagi, Estado do Paraná. Tese de Doutorado, Universidade Estadual Paulista, Rio Claro, SP.

Carmo, M.R.B., Andrade, A.L.P., Santos, G.A.S.D. \& Assis, M.A. 2012. Análise estrutural em relictos de cerrado no Parque Estadual do Guartelá, município de Tibagi, estado do Paraná, Brasil. Ciência Florestal, Santa Maria 22: 505-517.

Carmo, M.R.B., Luz, P.M., Lima, B.N.S. 2014. A vegetação rupestre. In: A.M. Gealh, M.S. Melo (orgs.). Rio São João, Carambeí, PR: fonte de vida, cuidados devidos, Universidade Estadual de Ponta Grossa, Ponta Grossa, pp. 101-110.

Carmo, F.F., Sousa, E., Fonseca, F.C., Ribeiro, L.C. \& Jacobi, C.M. 2007. Recrutamento pós-fogo em dois habitats de um campo rupestre ferruginoso (canga) na Serra da Moeda, MG. In: W. Deliti, E. Nascimento \& W. Mantovani (eds.). Anais do VIII Congresso de Ecologia do Brasil, Caxambu.

Cervi, A.C., Schwartz, E.A. \& Guimarães, O.A. 2003. Levantamento florístico de um campo do Primeiro Planalto Paranense, Curitiba, Paraná, Brasil. Sellowia 53-55: 29-50.

Cervi, A.C., Linsingen, L.V., Hatschbach, G. \& Ribas, O.S. 2007. A vegetação do Parque Estadual de Vila Velha, Município de Ponta Grossa, Paraná, Brasil. Boletim do Museu Botânico Municipal, Curitiba 69: $1-52$.

Chaghtai, S.M., Sadiq, A. \& Shah, S.H. 1985. Early succession on abandoned field in Peshawar, Pakistan. The Pakistan Journal of Forestry, v. 35, n. 2, pp. 59-69.

Conceição, A.A., Giulietti, A.M. \& Meirelles, S.T. 2007. Ilhas de vegetação em afloramentos de quartzito-arenito no Morro do Pai Inácio, Chapada Diamantina, Bahia, Brasil. Acta Botanica Brasilica 21: 335-347.

Coutinho, L.M. 1981. Aspectos ecológicos do fogo no cerrado. Revista Brasileira de Botânica, 4: 115-117.

Colwell, R.K. 2013. EstimateS: statistical estimation of species richness and shared species from samples. Version 9.1.0. Universidade de Connecticut, Connecticut. Disponível em http://viceroy.eeb.uconn.edu/estimates/ (acesso em 24-II-2016).

Curcio, G.C., Pires, D.R.J. \& Bognolla, I.A. 2004. Solos. In: Plano de manejo Parque Estadual de Vila Velha. Instituto Ambiental do Paraná, Curitiba, pp. 136-174.
Dalazoana, K. \& Moro, R.S. 2011. Riqueza específica em áreas de campo nativo impactadas por visitação turística e pastejo no Parque Nacional dos Campos Gerais, PR. Revista Floresta 41: 387-396.

Dalazoana, K., Silva, M.A. \& Moro, R.S. 2007. Comparação de três fisionomias de campo natural do Parque Estadual de Vila Velha, Ponta Grossa, PR. Revista Brasileira de Biociências 5: 675-677.

Daubenmire, R. 1968. Plant communities. Harper and Row, New York.

Dümig, A., Schad, P., Rumpel, C., Dignac, M.F. \& Kögel-Knabner, I. 2008. Araucaria forest expansion on grassland in the southern Brazilian highlands as revealed by $14 \mathrm{C}$ and $\delta 13 \mathrm{C}$ studies. Geoderma $145: 158-173$.

Longhi, S.J., Brun, E.J., Oliveira, D.M., Fialho, L.E.B., Wojciechowki, J.C. \& Vaccaro, S. 2005. Banco de sementes do solo em 3 fases sucessionais de uma floresta estacional Semidecidual em Santa Tereza, RS. Ciência Florestal, Santa Maria 15: 359-370.

Ferreira, P.M.A. \& Setubal, R.B. 2009. Florística e fitossociologia de um campo natural no município de Santo Antônio da Patrulha, Rio Grande do Sul, Brasil. Revista Brasileira de Biociências. Porto Alegre 7: 195-204.

Ferreira, A.P. \& Maranho, L.T. 2011. Avaliação da sucessão ecológica da estepe gramíneo lenhosa pouco alterada após fogo controlado e roçada no Parque Estadual de Vila Velha, PR, Brasil. In: O.T.B. Carpanezzi \& J.B. Campos, (orgs). Coletânea de Pesquisas: Parques Estaduais de Vila Velha, Cerrado e Guartelá. Curitiba: Instituto Ambiental do Paraná (IAP). pp. 36-45.

Flora do Brasil 2020 (em construção). 2016. Jardim Botânico do Rio de Janeiro. Disponível em http:// floradobrasil.jbrj.gov.br/ (acesso em 17-IV-2016).

Fidalgo, O. \& Bononi, V.L.R. 1984. Técnicas de coleta, preservação e herborização de material botânico. Instituto de Botânica, São Paulo.

Fidelis, A., Müller, S.C., Pillar, V.D. \& Pfadenhauer, J. 2007. Efeito do fogo na ecologia de populações de herbáceas e arbustos dos Campos Sulinos. Revista Brasileira de Biociências 5: 303-305.

Frost, P.G.H. \& Robertson, F. 1987. The ecological effects of fire in savannas. In: B.H. Walker (ed.). Determinants of Tropical Savannas. IRL Press, Oxford, pp. 93-140.

Hatschbach, G. \& Moreira-Filho, H. 1972. Catálogo florístico do Parque Estadual de Vila Velha (Estado do Paraná-Brasil). Boletim da Universidade Federal do Paraná 28: 1-53.

Heringer, I. \& Jacques, A.V.A. 2001. Adaptação das plantas ao fogo: enfoque na transição floresta - campo. Ciência Rural 31: 1-6.

IBGE - Instituto Brasileiro de Geografia e Estatística. 2012. Manual técnico da vegetação brasileira. Série Manuais Técnicos em Geociências. 2 ed. IBGE, Rio de Janeiro. 
Klein, R.M. \& Hatschbach, G. 1970/71. Fitofisionomia e notas complementares sobre o mapa fitogeográfico de Quero-Quero (Paraná). Boletim Paranaense de Geociências 28-29: 159-188.

Koeppen, W. 1948. Climatologia. Fundo de Cultura Econômica, México.

Kozera, C., Kuniyoshi, I.S., Galvão, F. \& Curcio, G.R. 2009. Composição florística de uma formação pioneira com Influência fluvial em Balsa Nova, PR, Brasil. Revista Floresta 39: 309-322.

Longhi-Wagner, H.M. 2003. Diversidade florística dos Campos sul brasileiros: Poaceae. In: Anais do $54^{\circ}$ Congresso Nacional de Botânica, Belém, pp. 117-120.

Maack, R. 1948. Notas preliminares sobre clima, solo e vegetação do Estado do Paraná. Arquivos de Biologia e Tecnologia 2: 102-200.

Maack, R. 1981. Geografia Física do Estado do Paraná. Livraria José Olympio, Rio de Janeiro.

Maack, R. 2012. Geografia Física do Estado do Paraná. 4 ed. Universidade Estadual de Ponta Grossa, Ponta Grossa, pp. 1- 526.

Medina, E. \& Huber, O. 1994. The role of biodiversity in the functioning of savanna ecosystems. In: O.T. Solbrig, H.M. Emden, P.G.W.J. Oordt (eds.). Biodiversity and Global Change. Cab International, Chicago.

Melo, M.S. 2006. Formas rochosas do Parque Estadual de Vila Velha. Universidade Estadual de Ponta Grossa, Ponta Grossa.

Melo, M.S., Moro, R.S. \& Guimarães, G.B. 2007. Os Campos Gerais do Paraná. In: M.S. Melo, R.S. Moro, \& G.B. Guimarães (eds.). Patrimônio Natural dos Campos Gerais do Paraná. Universidade Estadual de Ponta Grossa, Ponta Grossa, pp. 11-22.

Miranda, H.S., Sato, M.N., Neto, W.N. \& Aires, F.S. 2009. Fires in the Cerrado, the Brazilian savanna. In: M.A. Cochrane (ed.). Tropical fire ecology: climate change, land use, and ecosystem dynamics. SpringerPraxis, New York.

Moro, R.S. 2001. A Vegetação. In: C.H.M. Ditzel \& C.L.L. Sahr. Espaço e cultura: Ponta Grossa e os Campos Gerais. Universidade Estadual de Ponta Grossa, Ponta Grossa, pp. 408-503.

Moro, R.S. (org.). 2012. Biogeografia do Cerrado nos Campos Gerais. Editora UEPG, Ponta Grossa.

Moro, R.S. \& Carmo, M.R.B. 2007. A vegetação campestre nos Campos Gerais. In: M.S. Melo, R.S. Moro, \& G.B. Guimarães (eds.). Patrimônio Natural dos Campos Gerais do Paraná. Universidade Estadual de Ponta Grossa, Ponta Grossa, pp. 93-98.

Moro, R.S., Rocha, C.H., Takeda, J.M. \& Kaczmarech, R. 1996. Análise da vegetação nativa da bacia do Rio São Jorge. Publicatio UEPG: Ciências Biológicas e da Saúde, Ponta Grossa 2: 33-56.

Moro, R.S., Souza-Nogueira, M.K.F., Milan, E., Mioduski, J., Pereira, T.K. \& Moro, R.F. 2012. Grassland Vegetation of Pitangui River Valley, Southern Brazil. International Journal of Ecosystem 2: 161-170.
Müller, S.C., G.E. Overbeck., J. Pfadenhauer, \& V.D. Pillar. 2012. Woody species patterns at forest-grassland boundaries in southern Brazil. Flora, v. 207, 8: 586-598.

Nanuncio, V.N. \& Moro, R.S. 2008. O mosaico de vegetação remanescente em Piraí da Serra, Campos Gerais do Paraná: uma abordagem preliminar da fragmentaçãon natural da paisagem.Terr@Plural 2: $155-168$.

Oliveira, R.B. \& Godoy, S.A.P. 2007. Composição florística dos afloramentos rochosos do Morro do Forno, Altinópolis, São Paulo. Biota Neotropica 7: 39-47.

Oliveira, M.B. \& Maranho, L.T. 2011. Efeitos do fogo controlado e do roçado sobre a sucessão ecológica da Estepe Gramíneo-Lenhosa no Parque Estadual de Vila Velha, PR, Brasil, em área com avanço de vassoura (Bacharis spp.). In: O.T.B. Carpanezzi \& J.B. Campos. Coletânea de pesquisas: Parques Estaduais de Vila Velha, Cerrado e Guartelá. Instituto Ambiental do Paraná, Curitiba, pp. 46-55.

Overbeck, G.E., Müller, S. C., Pillar, V. D. P. \& Pfadenhauer, J. 2005. Fine-scale post-fire dynamics in southern Brazilian subtropical grassland. Journal of Vegetation Science. pp. 655-664.

Overbeck, G.E., Müller, S.C., Fidelis, A., Pfadenhauer, J., Pillar, V.D.P., Blanco, C., Boldrini, I.I., Both, R. \& Forneck, E. 2009. Os Campos Sulinos: um bioma negligenciado. In: V.D.P. Pillar, S.C. Müller, Z.M.S. Castilhos, A.V.A. Jacques (eds.). Campos Sulinos: conservação e uso sustentável da biodiversidade. Ministério do Meio Ambiente, Brasília, pp. 26-41.

SMA - Secretaria do Meio Ambiente. 1995. Lista vermelha de plantas ameaçadas de extinção no estado do Paraná. SEMA/GTZ, Curitiba.

Pichi-Sermolli, R.E.G. 1996. Authors of scientific names in Pteridophyta. Kew. Royal Botanic Gardens.

R Core Team. 2015. R: A language and environment for statistical computing. R Foundation for Statistical Computing. Disponível em https://www.R-project.org/ (acesso em 27-II-2016).

Ramos, A.F., Santana. A.C., Prieto, C.C. \& Matias, L.F. 2007. Mapeamento do uso da terra nos Campos Gerais. In: M.S. Melo, R.S. Moro \& G.B. Guimarães (eds.). Patrimônio Natural dos Campos Gerais do Paraná. Editora UEPG, Ponta Grossa, pp. 85-92.

Ritter, L.M.O., Almeida, C.G. \& Moro, R.S. 2007. Caracterização fitofisionômica dos fragmentos campestres com fácies de cerrado em Ponta Grossa, Paraná. Revista Brasileira de Biociências 5: 192-194.

Ritter, L.M.O., Ribeiro, M.C. \& Moro, R.S. 2010. Composição florística e fitofisionomia de remanescentes disjuntos de Cerrado nos Campos Gerais, PR, Brasil limite austral do bioma. Biota Neotropica 10: 379-414.

Selusniaki, M.A. 2013. Estrutura e composição florística dos campos naturais de São Luiz do Purunã, Balsa Nova - PR. Dissertação de Mestrado, Universidade Federal do Paraná, Curitiba. 
Setubal, R.B. \& Boldrini, I.I. 2012. Phytosociology and natural subtropical grassland communities on a granitic hill in southern Brazil. Rodriguésia 63: 513-524. Disponível em http://www.scielo.br/scielo. php?script=sci_arttext\&pid=S2175786020120003000 03\&lng=pt\&nrm=iso (acesso em 2-III-2016).

Souza, V.C. \& Lorenzi, H. 2008. Botânica Sistemática: guia ilustrado para identificação de famílias fenerógamas nativas e exóticas no Brasil, baseado no APG II. Instituto Plantarum, Nova Odessa.
Whelan, R.J. 1997. The Ecology of Fire. Cambridge University Press, Cambridge.

Ziller, S.R. \& Hatschbach, G. 1996. As formações vegetais do Parque Estadual do Guartelá, Tibagi, PR. SEMA/ IAP, Curitiba.

Ziller, S.R. \& Galvão, F. 2002. A degradação da estepe gramíneo lenhosa no Paraná por contaminação biológica de Pinus elliottii e P. taeda. Revista Floresta 32: 41-47.

Zuur, A.K., Ieno, E.N. \& Smith, G.M. 2007. Analyzing Ecological Data. Springer-Verlag New York, New York. 